\title{
Pacific
}

Journal of

Mathematics

\section{SEQUENCES OF OPEN RIEMANNIAN MANIFOLDS WITH BOUNDARY}

RAQuel Perales AND Christina SoRMAni 


\title{
SEQUENCES OF OPEN RIEMANNIAN MANIFOLDS WITH BOUNDARY
}

\author{
RAQuel PERAles AND Christina SORMAni
}

\begin{abstract}
We consider sequences of open Riemannian manifolds with boundary that have no regularity conditions on the boundary. To define a reasonable notion of a limit of such a sequence, we examine $\delta$-inner regions, that avoid the boundary by a distance $\delta$. We prove Gromov-Hausdorff compactness theorems for sequences of these $\delta$-inner regions. We then build "glued limit spaces" out of the Gromov-Hausdorff limits of $\delta$-inner regions and study the properties of these glued limit spaces. Our main applications assume the sequence is noncollapsing and has nonnegative Ricci curvature. We include open questions.
\end{abstract}

\section{Introduction}

Recall that Gromov's Ricci compactness theorem states that a sequence of compact Riemannian manifolds with nonnegative Ricci curvature and a uniform upper bound on diameter has a subsequence that converges in the Gromov-Hausdorff sense to a metric space [8]. When the sequence of manifolds is noncollapsing, GromovHausdorff limit spaces have a variety of properties, particularly restrictions on their metrics, their Hausdorff measures, and their topologies. These properties were proven by Cheeger, Colding, Naber, Wei and the second author $[3 ; 4 ; 13 ; 5]$.

Here we consider an open Riemannian manifold $\left(M^{m}, g\right)$ endowed with the length metric $d_{M}$, as in (3). We define the boundary to be

$$
\partial M=\bar{M} \backslash M,
$$

where $\bar{M}$ is the metric completion of $M$. For example, $\left(M^{m}, g\right)$ may be a smooth manifold with boundary. However, we do not require any smoothness conditions on this boundary.

First observe that Gromov's Ricci compactness theorem does not hold for precompact open manifolds with boundary that have a uniform upper bound on diameter, even if they are flat and two-dimensional:

Perales is a doctoral student at Stony Brook. Sormani's research is partially supported by NSF DMS 10060059.

MSC2010: 53C23.

Keywords: Gromov-Hausdorff, manifold, boundary. 


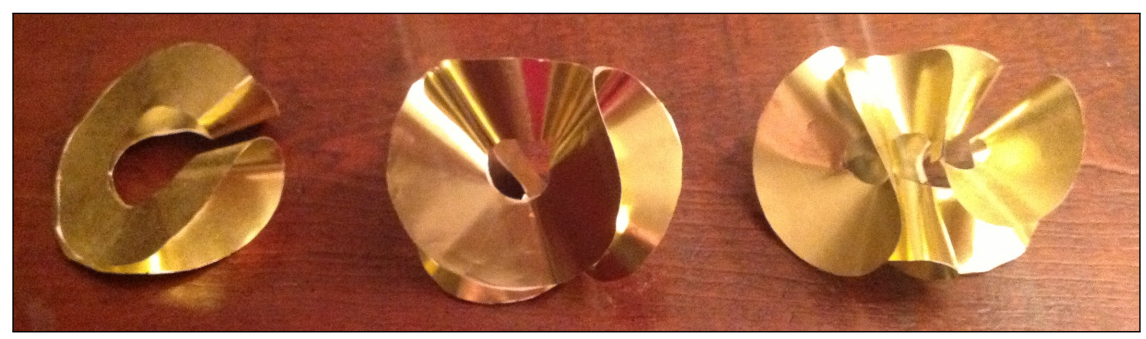

Figure 1. Models of Example 1.1: $M_{2}, M_{3}, M_{4}, \ldots$

Example 1.1. The $j$-fold covering spaces $M_{j}$ of the annuli $\operatorname{Ann}_{0}(1 / j, 1) \subset \mathbb{E}^{2}$, depicted in Figure 1, are flat surfaces such that

$$
\operatorname{Diam}\left(M_{j}\right) \leq 2+\pi \quad \text { and } \quad \operatorname{Vol}\left(M_{j}\right)=j\left(\pi-\pi(1 / j)^{2}\right) .
$$

See Remark 5.5 for the proof that there is no subsequence of these spaces with a Gromov-Hausdorff limit.

Assuming both a uniform upper bound on volume and diameter, we still do not have Gromov-Hausdorff compactness:

Example 1.2. The smooth regions $M_{j} \subset \mathbb{E}^{2}$ with many spikes, depicted in Figure 2, have no subsequence with a Gromov-Hausdorff limit. See Example 2.13 for details.

Compactness theorems for sequences of Riemannian manifolds with boundary, assuming curvature controls on the boundary, have been proven by Kodani [11], Anderson, Katsuda, Kurylev, Lassas, and Taylor [1], Wong [14] and Knox [10]. A survey of these results has been written by the first author [12]. Since we do not wish to assume the boundary is smooth, we prove compactness theorems for regions which avoid the boundary (Theorem 1.4). We then glue together the limits of these regions (Theorem 6.3) and prove that these glued limit spaces have nice properties (Theorem 8.8).

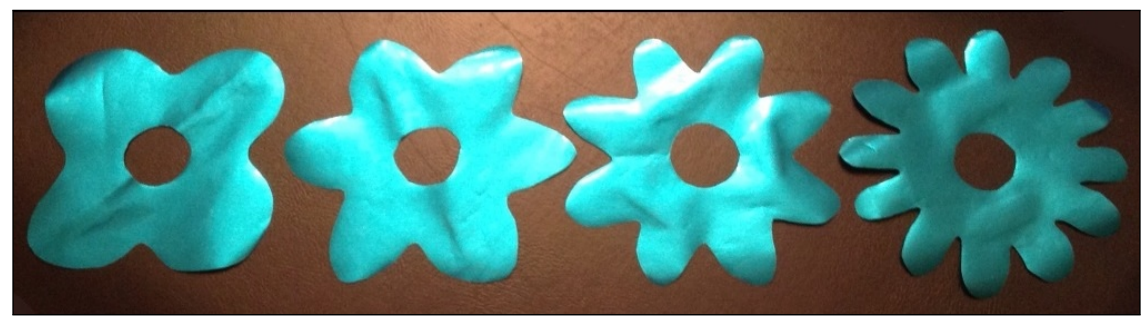

Figure 2. Models of Example 1.2: $M_{4}, M_{6}, M_{8}, M_{12}, \ldots$ 
Definition 1.3. Given an open Riemannian manifold $\left(M, g_{M}\right)$ and $\delta>0$, we define the $\delta$-inner region as

$$
M^{\delta}=\left\{x \in M: d_{M}(x, \partial M)>\delta\right\}
$$

where $\partial M$ is defined as in (1),

$$
d_{M}(x, y):=\inf \left\{L_{g}(C): C:[0,1] \rightarrow M, C(0)=x, C(1)=y\right\},
$$

and

$$
L_{g}(C)=\int_{0}^{1} g\left(C^{\prime}(t), C^{\prime}(t)\right) d t
$$

There are two metrics on the $\delta$-inner region $M^{\delta}$ : the restricted metric $d_{M}$ and the induced length metric

$$
d_{M^{\delta}}(x, y):=\inf \left\{L_{g}(C): C:[0,1] \rightarrow M^{\delta}, C(0)=x, C(1)=y\right\} .
$$

Note that $d_{M^{\delta}}$ is only defined between points in the same connected component of $M^{\delta}$. The intrinsic diameter

$$
\operatorname{Diam}\left(M^{\delta}, d_{M^{\delta}}\right)=\sup \left\{d_{M^{\delta}}(x, y): x, y \in M^{\delta}\right\}
$$

will be infinite if $M^{\delta}$ is not connected by rectifiable paths.

Theorem 1.4. Given $m \in \mathbb{N}, \delta>0, D>0, V>0$, and $\theta>0$, let $\mu_{\theta}^{m, \delta, D, V}$ be the class of open m-dimensional Riemannian manifolds $M$ with boundary with nonnegative Ricci curvature, $\operatorname{Vol}(M) \leq V$, and

$$
\operatorname{Diam}\left(M^{\delta}, d_{M^{\delta}}\right) \leq D
$$

that are noncollapsing at a point, in the sense that

$$
\operatorname{Vol}\left(B_{q}(\delta)\right) \geq \theta \delta^{m} \quad \text { for some } q \in M^{\delta} .
$$

If $\left(M_{j}, g_{j}\right) \subset M_{\theta}^{m, \delta, D, V}$, there is a subsequence $\left(M_{j_{k}}^{\delta}, d_{M_{j_{k}}}\right)$ such that the metric completions with the restricted metric $d_{M_{j}}$ converge in the Gromov-Hausdorff sense to a metric space $\left(Y^{\delta}, d\right)$.

Example 1.2 satisfies the conditions of this theorem, demonstrating why we can only obtain Gromov-Hausdorff convergence of the $M_{j}^{\delta}$ instead of the $M_{j}$ themselves. The $M_{j}^{\delta}$ of Example 1.1 do not have Gromov-Hausdorff convergent subsequences (see Remark 5.5), demonstrating the necessity of the hypothesis requiring an upper bound on the volume. In Theorem 5.2, we remove the intrinsic diameter condition (5) and the noncollapsing condition (6), and assume conditions on closed geodesics and constant sectional curvature instead. 
Theorem 1.4 and Theorem 5.2 are proved in Section 5. We start by reviewing Gromov-Hausdorff convergence in Section 2. In Sections 3 and 4 we study the limits of inner regions in sequences of manifolds that have Gromov-Hausdorff limits. See in particular Theorem 4.1. These sections contain many examples.

In Section 6 we define glued limit spaces for any sequence of open Riemannian manifolds $\left(M_{j}, g_{j}\right)$, assuming that for all $\delta>0$, the $\left(M_{j}^{\delta}, d_{j}\right)$ converge in the Gromov-Hausdorff sense to a metric space $\left(Y^{\delta}, d_{\delta}\right)$. We build a "glued limit space" $\left(Y, d_{Y}\right)$ from these $Y^{\delta}$ in Theorem 6.1 and Theorem 6.3. The metric completion of a glued limit space is called a "completed glued limit space."

Note that this glued limit space may exist even when $\left(M_{j}, d_{j}\right)$ has no GromovHausdorff limit, as in Example 2.13 (see Remark 6.10). The glued limit may not be precompact even when one has a sequence of flat Riemannian manifolds with boundary (Examples 6.11 and 6.12).

In general the completed glued limit space of a sequence of $M_{j}$ need not be unique (Example 6.16). However, if the $\left(M_{j}, d_{M_{j}}\right)$ have a Gromov-Hausdorff limit $\left(X, d_{X}\right)$, then the completed glued limit space is unique and is embedded isometrically into $X$ (Theorem 6.6). The completed glued limit space need not be isometric to the Gromov-Hausdorff limit (Example 4.10) even when the $\left(M_{j}, g_{j}\right)$ are regions in the Euclidean plane satisfying all the hypothesis of Theorem 1.4 (Remark 6.7). Intuitively, regions which collapse relative to the boundary disappear, while regions which collapse that lie far from the boundary need not disappear.

In Section 7 we apply Theorems 5.2 and 1.4 to construct glued limit spaces for sequences of manifolds with curvature bounds (Theorems 7.1 and 7.4). In Section 8 we explore the properties of these glued limit spaces. First we present an example where the curvature bounds in the sequence of manifolds is lost in the Gromov-Hausdorff limit (Example 8.1). Then we prove Proposition 8.4 concerning glued limits of manifolds with constant sectional curvature. We close with Theorem 8.8, proving that glued limits constructed under the conditions of Theorem 1.4 have Hausdorff dimension $m$, Hausdorff measure at most $V$, and positive density everywhere. This final theorem is proved using Theorem 8.3, which proves certain balls in glued limit spaces are the Gromov-Hausdorff limits of nice balls in the open manifolds, combined with the Bishop-Gromov volume comparison theorem [8] and Colding's volume convergence theorem [4].

Throughout the paper we state open questions at 6.14, 8.6, 8.7, 8.10, and 8.9. The first author is in the process of proving Open question 8.10 as part of her doctoral dissertation. Please contact us if you would like to work on one of the other open questions or if you are interested in extending our theorems to the setting where the sequence has a negative uniform lower Ricci curvature bound or is allowed to collapse. 


\section{Background}

Here we review Gromov-Hausdorff convergence and Gromov's compactness theorem [8]. A good resource for this material is [2].

2A. Hausdorff convergence. In [8], Gromov defined the Gromov-Hausdorff distance between pairs of compact metric spaces. We review this definition here.

Definition 2.1 (Hausdorff). The Hausdorff distance between two compact subsets $A_{1}, A_{2}$ of a metric space $Z$ with metric $d_{Z}$ is defined as

$$
d_{H}^{Z}\left(A_{1}, A_{2}\right)=\inf \left\{r: A_{1} \subset T_{r}\left(A_{2}\right), A_{2} \subset T_{r}\left(A_{1}\right)\right\},
$$

where the tubular neighborhood $T_{r}(A)$ is the set $T_{r}(A)=\left\{x \in Z: d_{Z}(x, A)<r\right\}$.

Observe that if one has a sequence of compact subsets $A_{j} \subset Z$ such that $d_{H}\left(A_{j}, A_{\infty}\right) \rightarrow 0$, then for all $a \in A_{\infty}$ there exists $a_{j} \in A_{j}$ such that $\lim _{j \rightarrow \infty} a_{j}=a$.

Lemma 2.2. Suppose $A_{j} \subset Z$ are compact, $d_{H}^{Z}\left(A_{j}, A_{\infty}\right)=h_{j} \rightarrow 0, a_{j} \in A_{j}$ and $a_{\infty} \in A_{\infty}$ such that $d_{Z}\left(a_{j}, a_{\infty}\right)=\delta_{j} \rightarrow 0$. Then for all $r>0$ there exist $r_{j}=r+\delta_{j}+h_{j} \rightarrow r$ such that the closed balls converge:

$$
d_{H}^{Z}\left(\bar{B}_{a_{j}}\left(r_{j}\right) \cap A_{j}, \bar{B}_{a_{\infty}}(r) \cap A_{\infty}\right) \rightarrow 0 .
$$

Here we are not assuming that $A_{\infty}$ or $A_{j}$ are length spaces. For completeness of exposition we include the proof of this well-known lemma:

Proof. Suppose $x \in \bar{B}_{a_{\infty}}(r) \cap A_{\infty}$; then $d_{Z}\left(x, a_{\infty}\right) \leq r$ and $x \in A_{\infty} \subset T_{h_{j}}\left(A_{j}\right)$. So there exists $y_{j} \in A_{j}$ such that $d_{Z}\left(x, y_{j}\right)<h_{j}$. By the triangle inequality,

$$
d\left(y_{j}, a_{j}\right) \leq d\left(y_{j}, x\right)+d\left(x, a_{\infty}\right)+d\left(a_{\infty}, a_{j}\right) \leq h_{j}+r+\delta_{j}=r_{j} .
$$

Thus

$$
\bar{B}_{a_{\infty}}(r) \cap A_{\infty} \subset T_{h_{j}}\left(\bar{B}_{a_{j}}\left(r_{j}\right) \cap A_{j}\right) .
$$

Now we need only show that for all $\varepsilon>0$ the following inclusion holds for all sufficiently large $j$ :

$$
\bar{B}_{a_{j}}\left(r_{j}\right) \cap A_{j} \subset T_{\varepsilon}\left(\bar{B}_{a_{\infty}}(r) \cap A_{\infty}\right) .
$$

Suppose not. Then there exist $\varepsilon_{0}>0$, a subsequence $j \rightarrow \infty$ and elements

$$
x_{j} \in\left(\bar{B}_{a_{j}}\left(r_{j}\right) \cap A_{j}\right) \backslash T_{\varepsilon_{0}}\left(\bar{B}_{a_{\infty}}(r) \cap A_{\infty}\right) .
$$

Since $Z$ is compact and $T_{\varepsilon_{0}}\left(\bar{B}_{a_{\infty}}(r) \cap A_{\infty}\right)$ is open, a subsequence of the $x_{j}$ converges to some

$$
x_{\infty} \notin T_{\varepsilon_{0}}\left(\bar{B}_{a_{\infty}}(r) \cap A_{\infty}\right) .
$$

Since $d\left(x_{j}, a_{j}\right) \leq r_{j}$, we have $d\left(x_{\infty}, a_{\infty}\right) \leq r$. Since $x_{j} \in A_{j}$, there exists $y_{j} \in A_{\infty}$ 
such that $d\left(x_{j}, y_{j}\right)<h_{j}$. By the triangle inequality,

$$
y_{j} \in B_{a_{\infty}}\left(r+h_{j}\right) \cap A_{\infty} .
$$

Observe that, for our subsequence, $y_{j} \rightarrow x_{\infty}$; thus

$$
x_{\infty} \in \bar{B}_{a_{\infty}}(r) \cap A_{\infty} \subset T_{\varepsilon_{0}}\left(\bar{B}_{a_{\infty}}(r) \cap A_{\infty}\right),
$$

which is a contradiction.

\section{B. Gromov-Hausdorff convergence.}

Definition 2.3. An isometric embedding $\varphi:\left(X, d_{X}\right) \rightarrow\left(Z, d_{Z}\right)$ between metric spaces is a mapping which preserves distances:

$$
d_{Z}\left(\varphi\left(x_{1}\right), \varphi\left(x_{2}\right)\right)=d_{X}\left(x_{1}, x_{2}\right) .
$$

Definition 2.4 (Gromov). The Gromov-Hausdorff distance between a pair of compact metric spaces, $\left(X_{1}, d_{X_{1}}\right)$ and $\left(X_{2}, d_{X_{2}}\right)$, is defined as

$$
d_{\mathrm{GH}}\left(\left(X_{1}, d_{X_{1}}\right),\left(X_{2}, d_{X_{2}}\right)\right)=\inf \left\{d_{Z}\left(\varphi_{1}\left(X_{1}\right), \varphi_{2}\left(X_{2}\right)\right): \varphi_{i}: X_{i} \rightarrow Z\right\}
$$

where the infimum is taken over all isometric embeddings $\varphi_{i}: X_{i} \rightarrow Z$ and all metric spaces $Z$.

Gromov proved that the Gromov-Hausdorff distance is a distance on the space of compact metric spaces. When studying metric spaces $X_{i}$ which are only precompact, one takes the metric completions $\bar{X}_{i}$ before comparing such spaces using the Gromov-Hausdorff distance:

Definition 2.5. Given a precompact metric space $\left(X, d_{X}\right)$, the metric completion $\left(\bar{X}, d_{X}\right)$ consists of equivalence classes of Cauchy sequences $\left\{x_{1}, x_{2}, x_{3}, \ldots\right\}$ in $X$, where

$$
d_{X}\left(\left\{x_{j}\right\},\left\{y_{j}\right\}\right)=\lim _{j \rightarrow \infty} d_{X}\left(x_{j}, y_{j}\right),
$$

and two Cauchy sequences are equivalent if the distance between them is 0 . There is an isometric embedding

$$
\varphi: X \rightarrow \bar{X} \quad \text { given by } \quad \varphi(x)=\{x, x, x, \ldots\} .
$$

In this paper we define the boundary of an open metric space to be

$$
\partial X=\bar{X} \backslash X .
$$

When $M$ is a smooth Riemannian manifold with boundary, then this notion of boundary agrees with the standard notion of boundary. However, if $M$ is a smooth Riemannian manifold with a singular point removed, then the boundary in our setting is just the missing singular point. 
2C. Lattices and Gromov-Hausdorff convergence. One technique that can be applied to produce amazingly complicated Gromov-Hausdorff limits from surfaces is the construction of lattices. The basic, well-known lemma is as follows:

Lemma 2.6. Let $X=\left[a_{1}, b_{1}\right] \times \cdots \times\left[a_{k}, b_{k}\right]$ with the taxi product metric

$$
d_{X}\left(\left(x_{1}, \ldots, x_{k}\right),\left(y_{1}, \ldots, y_{k}\right)\right)=\sum_{i=1}^{k}\left|x_{i}-y_{i}\right| .
$$

Then for any $\varepsilon>0$ there exists a 2-dimensional manifold $M_{\varepsilon}$ such that

$$
d_{\mathrm{GH}}\left(M_{\varepsilon}, X\right)<\varepsilon .
$$

The classic application of this lemma is to construct a Gromov-Hausdorff limit of Riemannian surfaces which is infinite-dimensional:

Example 2.7. Let $X_{j}=[0,1] \times\left[0, \frac{1}{2}\right] \times \cdots \times\left[0,\left(\frac{1}{2}\right)^{j}\right]$ with the taxi metric, and let

$$
X=[0,1] \times\left[0, \frac{1}{2}\right] \times \cdots \times\left[0,\left(\frac{1}{2}\right)^{j}\right] \times \cdots
$$

be the infinite-dimensional space also with the taxi metric

Then

$$
d_{X}\left(\left(x_{1}, x_{2}, \ldots\right),\left(y_{1}, y_{2}, \ldots\right)\right)=\sum_{i=1}^{\infty}\left|x_{i}-y_{i}\right| .
$$

$$
d_{\mathrm{GH}}\left(X_{k}, X\right) \leq \sum_{j=k+1}^{\infty}\left(\frac{1}{2}\right)^{j}=\left(\frac{1}{2}\right)^{k} \rightarrow 0 .
$$

Thus, by Lemma 2.6, we have a sequence of surfaces $M_{k}$ converging to $X$ as well.

Since we are interested in manifolds with boundary, we will prove a stronger version of Lemma 2.6 that can be applied to produce examples later in the paper.

Proposition 2.8. Suppose that $X=\left[a_{1}, b_{1}\right] \times \cdots \times\left[a_{k}, b_{k}\right]$ with the taxi product metric, and let $A \subset \partial X$ (possibly empty). Then for any $\varepsilon>0$, there exists an open Riemannian surface $M$ with boundary $\partial M$ (possibly empty) such that

$$
d_{\mathrm{GH}}(M, X)<\varepsilon \quad \text { and } \quad d_{\mathrm{GH}}(\partial M, A)<\varepsilon .
$$

Suppose we have a collection of $X_{k}$ and $A_{k} \subset \partial X_{k}$ as above, with subsets $B_{k} \subset X_{k}$ and isometric embeddings $\psi_{k}: B_{k+1} \rightarrow B_{k}$, and we glue $X=X_{1} \sqcup X_{2} \sqcup \cdots \sqcup X_{k}$ via these isometric embeddings, and set $A=\bigcup A_{k} \subset X$. Then for any $\varepsilon>0$ we have an open Riemannian surface $M$ with boundary $\partial M$ (possible empty) such that

$$
d_{\mathrm{GH}}(M, X)<\varepsilon \quad \text { and } \quad d_{\mathrm{GH}}(\partial M, A)<\varepsilon .
$$

In fact, for any $\delta>0$, using the restricted distances, we have

$$
d_{\mathrm{GH}}\left(\left(M \backslash T_{\delta}(\partial M), d_{M}\right),\left(X \backslash T_{\delta}(A), d_{X}\right)\right)<\varepsilon .
$$


Proof. For the first part, we take a lattice $Y_{\varepsilon}^{\prime} \subset Y_{\varepsilon} \subset X$ such that $X \subset T_{\varepsilon / 2}\left(Y_{\varepsilon}\right)$. Here we use $Y_{\varepsilon}^{\prime}$ to denote the points and $Y_{\varepsilon}$ to include 1-dimensional edges between the points in the lattice. Observe that $d_{Y_{\varepsilon}}\left(y_{1}, y_{2}\right)=d_{X}\left(y_{1}, y_{2}\right)$ because we are using the taxi norm. Let $A_{\varepsilon} \subset Y_{\varepsilon}^{\prime}$ be chosen such that $A_{\varepsilon} \subset T_{\varepsilon / 2}(A)$, so

$$
d_{\mathrm{GH}}\left(Y_{\varepsilon}, X\right)<\frac{\varepsilon}{2} \quad \text { and } \quad d_{\mathrm{GH}}\left(A_{\varepsilon}, A\right)<\frac{\varepsilon}{2} .
$$

Note that we may now view $Y_{\varepsilon}$ as a graph. For example, if $X=[0,5] \times[0,6]$, $A=[0,5] \times\{6\}$, and $\varepsilon=1$, then the left side of Figure 3 is the graph $Y_{\varepsilon}$, with $A_{\varepsilon}$ depicted in red.

Next we construct a smooth surface $M$ by replacing the lattice points in $A_{\varepsilon} \subset Y_{\varepsilon}^{\prime}$ by small hemispheres of diameter $\ll \varepsilon$ and lattice points in $Y_{\varepsilon}^{\prime} \backslash A_{\varepsilon}$ by small spheres of diameter $\ll \varepsilon$. We replace the line segments in $Y_{\varepsilon}$ by arbitrarily thin cylinders of the same length, small enough that we can glue them to their corresponding spheres, smoothly replacing disjoint balls in those spheres or hemispheres. This creates a smooth manifold $M$ such that $\partial M$ is a union of the boundaries of the hemispheres, and such that

$$
d_{\mathrm{GH}}\left(Y_{\varepsilon}, M\right)<\frac{\varepsilon}{2} \quad \text { and } \quad d_{\mathrm{GH}}\left(A_{\varepsilon}, \partial M\right)<\frac{\varepsilon}{2} .
$$

See the right side of Figure 3, where $M^{2}$ is depicted in gray and $\partial M^{2}$ is in red. This completes the first claim in the proposition.

To complete the rest, we take $M_{k}$ consisting of tubes joined at spheres and hemispheres close to $X_{k}$, as above, such that

$$
d_{\mathrm{GH}}\left(X_{k}, M_{k}\right)<\frac{\varepsilon}{k} \quad \text { and } \quad d_{\mathrm{GH}}\left(A_{k}, \partial M_{k}\right)<\frac{\varepsilon}{k} .
$$

Note that in the construction above we could have created $B_{k}^{\prime} \subset Y_{k}^{\prime}$ corresponding to $B_{k}$. We have $\varepsilon /(2 k)$ almost distance-preserving maps $\psi_{k}^{\prime}: B_{k+1}^{\prime} \rightarrow B_{k}^{\prime}$. So now we glue together the $M_{k}$ to form $M$ as follows. If $b \in B_{k}^{\prime}$ maps to $\psi_{k}^{\prime}(b) \in B_{k}^{\prime}$,
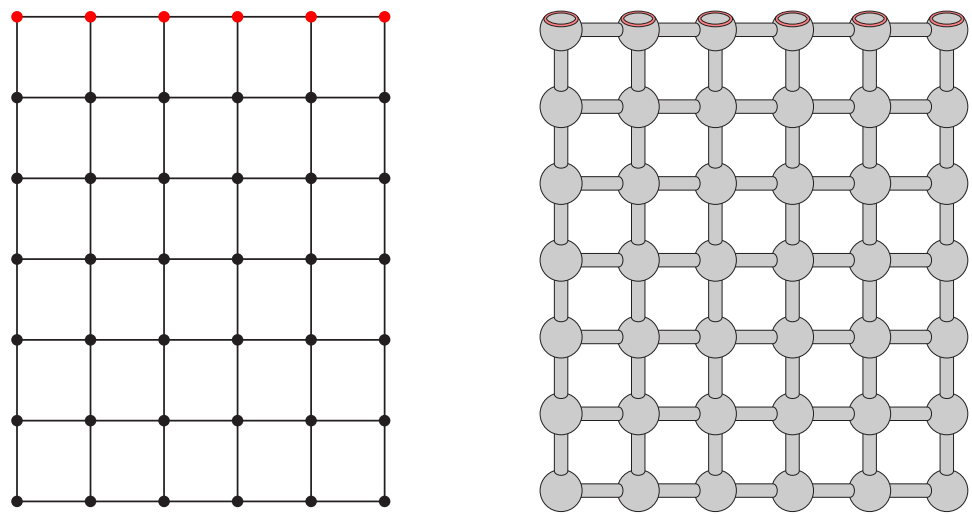

Figure 3. $A_{\varepsilon} \subset Y_{\varepsilon}$ and $\partial M \subset M$ as in the proof of Proposition 2.8. 
we connect the sphere or hemisphere corresponding to $b$ in $M_{k}$ to a sphere or hemisphere corresponding to $\psi_{k}(b)$ in $M_{k+1}$ by a very short, very thin tube.

2D. Review of Gromov's compactness theorem. In [8], Gromov proved a compactness theorem for sequences of compact metric spaces. We review this theorem and related propositions here.

Theorem 2.9 (Gromov). Given $D>0$ and a function $N:(0, D] \rightarrow \mathbb{N}$, we define the collection $\mu^{D, N}$ of compact metric spaces $\left(X, d_{X}\right)$ with diameter $\leq D$ that can be covered by $N(\epsilon)$ balls of radius $\epsilon>0$ :

$$
X \subset \bigcup_{i=1}^{N(\epsilon)} B_{x_{i}}(\epsilon)
$$

This collection $\mu^{D, N}$ is compact with respect to the Gromov-Hausdorff distance.

It is standard to determine whether a metric space lies in such a compact collection by examining maximal collections of disjoint balls:

Proposition 2.10. Given a metric space $\left(X, d_{X}\right)$, let $N$ be the maximum number of pairwise disjoint balls of radius $\epsilon / 2$ that can lie in $X$. Then the minimum number of balls of radius $\epsilon$ required to cover $X$ is at most $N$.

Proof. Let $\left\{B_{x_{i}}(\epsilon / 2): i=1, \ldots, N\right\}$ be a maximal collection of pairwise disjoint balls of radius $\epsilon / 2$. Let $x \in X$. Then $B_{x_{i}}(\epsilon / 2) \cap B_{x}(\epsilon / 2)$ is nonempty for some $i \in\{1, \ldots, N\}$. Thus $d_{X}\left(x, x_{i}\right)<\epsilon$, and

$$
X \subset \bigcup_{i=1}^{N} B_{x_{i}}(\epsilon)
$$

In a Riemannian manifold or metric measure space, the volumes of balls may thus be applied to determine the function $N$.

Proposition 2.11. If there exists $\Theta>0$ such that

$$
\operatorname{Vol}\left(B_{p}(\epsilon)\right) / \operatorname{Vol}(M) \geq \Theta
$$

then the maximum number of disjoint balls of radius $\epsilon$ is at most $1 / \Theta$.

Proof. We have

$$
\operatorname{Vol}(M) \geq \sum_{i=1}^{N} \operatorname{Vol}\left(B_{x_{i}}(\epsilon)\right) \geq \sum_{i=1}^{N} \Theta \operatorname{Vol}(M)=N \Theta \operatorname{Vol}(M) .
$$


Gromov applies his compactness theorem in conjunction with these propositions to study the compactness of sequences of compact Riemannian manifolds for which one is able to control the volumes of balls. We will apply the same idea to study sequences of metric completions of open manifolds.

One of the beauties of Gromov's compactness theorem is that the converse holds as well:

Theorem 2.12 (Gromov). Suppose $\left(X_{j}, d_{j}\right)$ are compact metric spaces. Suppose that there exists $\epsilon_{0}>0$ such that $X_{j}$ contains at least $j$ disjoint balls of radius $\epsilon_{0}$. Then no subsequence of the $X_{j}$ has a Gromov-Hausdorff limit.

In particular, if $\left(X_{j}, d_{X_{j}}\right) \stackrel{\mathrm{GH}}{\longrightarrow}\left(X, d_{X}\right)$, then they have a uniform upper bound on diameter. Nor can they have many spikes, as in the following example:

Example 2.13. Let

$$
M_{j}=\left\{(\theta, r): \theta \in S^{1}, r \in(1,3+\cos (j \theta))\right\}
$$

with metric $g_{j}=d r^{2}+r^{2} d \theta^{2}$. Then $\operatorname{Vol}\left(M_{j}\right) \leq \pi 4^{2}, \operatorname{Diam}\left(M_{j}\right) \leq 3+\pi+3$, and $M_{j}$ has 0 sectional curvature.

Observe that in $M_{j}$ the balls of radius 1 about $(2 \pi k / j, 3)$ are disjoint because paths between these points in $M_{j}$ must reach within $r \leq 2$ between the spikes and so have length at least $2(3-2)$. Thus, there are $j$ disjoint balls of radius 1 in $M_{j}$, and no subsequence of the metric completions of the $M_{j}$ converges in the Gromov-Hausdorff sense.

Example 2.14. Let

$$
X_{j}=([0,1] \times[0,1]) \sqcup\left([0,1] \times\left[0, \frac{1}{2}\right]\right) \sqcup \cdots \sqcup\left([0,1] \times\left[0,\left(\frac{1}{2}\right)^{j}\right]\right)
$$

be a disjoint union of spaces with taxicab metrics glued via the map $\psi(0, y)=(0, y)$. Then $X_{j}$ has no Gromov-Hausdorff convergent subsequence, because it has $j$ disjoint balls of radius 1 about the points $(1,0)$. If we take the surfaces $M_{j}$ as constructed in Proposition 2.8, such that

$$
d_{\mathrm{GH}}\left(M_{j}, X_{j}\right) \rightarrow 0,
$$

they also have no Gromov-Hausdorff convergent subsequence.

In a later paper, Gromov proved the following useful theorem [7, page 65] by defining an appropriate compact metric space and applying Theorem 2.16.

Theorem 2.15 (Gromov). If one has a sequence of compact metric spaces $\left(X_{j}, d_{X_{j}}\right)$ such that $\left(X_{j}, d_{X_{j}}\right) \stackrel{\mathrm{GH}}{\longrightarrow}\left(X_{\infty}, d_{X_{\infty}}\right)$, then there exists a common compact metric space $Z$ and isometric embeddings $\varphi_{j}:\left(X_{j}, d_{X_{j}}\right) \rightarrow\left(Z, d_{Z}\right)$ such that $d_{H}\left(\varphi_{j}\left(X_{j}\right), \varphi_{\infty}\left(X_{\infty}\right)\right) \rightarrow 0$. 
Theorem 2.16 (Blaschke). If $Z$ is a compact metric space then every sequence of closed subsets of $Z$ has a subsequence that converges in the Hausdorff sense to a closed subset.

Theorem 2.15 implies the Gromov-Hausdorff Arzelà-Ascoli theorem:

Theorem 2.17 (Gromov). Assume $X_{j} \stackrel{\mathrm{GH}}{\longrightarrow} X, Y_{j} \stackrel{\mathrm{GH}}{\longrightarrow} Y$, and let $f_{j}: X_{j} \rightarrow Y_{j}$ be an equicontinuous sequence; i.e., for all $\epsilon>0$ there exists $\delta_{\epsilon}>0$ such that

$$
d_{X_{j}}(p, q)<\delta_{\epsilon} \Longrightarrow d_{Y_{j}}\left(f_{j}(p), f_{j}(q)\right)<\epsilon .
$$

Then there is a subsequence with a continuous limit function $f: X \rightarrow Y$. If the $f_{j}$ are isometric embeddings, then so is $f$.

In particular, if the $X_{j}$ are geodesic spaces, then so is the limit space [8].

2E. Gromov's Ricci compactness theorem. In this section we review Gromov's Ricci compactness theorem, which is based on the Bishop-Gromov volume comparison theorem [8]:

Theorem 2.18 (Bishop-Gromov). If $M$ is an m-dimensional Riemannian manifold with boundary having nonnegative Ricci curvature, and $B_{p}(R) \subset M^{m}$ does not reach the boundary, then for all $r \in(0, R)$ we have

$$
\frac{\operatorname{Vol}\left(B_{p}(r)\right)}{\operatorname{Vol}\left(B_{p}(R)\right)} \geq\left(\frac{r}{R}\right)^{m} .
$$

Gromov's Ricci compactness theorem was originally stated for compact manifolds without boundary:

Theorem 2.19 (Gromov). Let $m \in \mathbb{N}, D>0$ and let $\mu^{m, D}$ be the class of compact $m$-dimensional Riemannian manifolds $M$ with nonnegative Ricci curvature and $\operatorname{Diam}(M) \leq D$. Here the manifolds do not have boundary. Then $\mathcal{M}^{m, D}$ is precompact with respect to the Gromov-Hausdorff distance.

In fact, Gromov's compactness theorem has a commonly used version applied to balls, which we state as follows:

Theorem 2.20 (Gromov). Let $m \in \mathbb{N}, D>0$ and let $\mathcal{M}^{m}$ be the class of compact $m$-dimensional Riemannian manifolds $M$ with nonnegative Ricci curvature. If $M_{j} \in \mathcal{M}^{m}$ and $p_{j} \in M_{j}$ such that $d\left(p_{j}, \partial M_{j}\right)>D$, there exists a subsequence such that $\left(B_{p_{j}}(D / 3), d_{M_{j}}\right)$ converges in the Gromov-Hausdorff distance.

For completeness of exposition we show how Gromov's original proof implies Theorem 2.20. 
Proof. Let $q \in B_{p_{j}}(D / 3)$. Then $B_{p_{j}}(D / 3) \subset B_{q}(2 D / 3) \subset B_{p_{j}}(D)$ does not reach the boundary of $M_{j}$, so we may apply the Bishop-Gromov volume comparison theorem to see that

$$
\begin{aligned}
\frac{\operatorname{Vol}\left(B_{q}(r)\right)}{\operatorname{Vol}\left(B_{p_{j}}(D / 3)\right)} & \geq \frac{r^{m}}{(2 D / 3)^{m}} \frac{\operatorname{Vol}\left(B_{q}(2 D / 3)\right)}{\operatorname{Vol}\left(B_{p_{j}}(D / 3)\right)} \\
& \geq \frac{r^{m}}{(2 D / 3)^{m}} \frac{\operatorname{Vol}\left(B_{p_{j}}(D / 3)\right)}{\operatorname{Vol}\left(B_{p_{j}}(D / 3)\right)}=\frac{(3 r)^{m}}{(2 D)^{m}} .
\end{aligned}
$$

So now we may apply Proposition 2.11 to complete the proof.

2F. Volume convergence theorems. In [4], Colding proved the following volume convergence theorem:

Theorem 2.21 (Colding). Let $M_{j}^{m}$ be complete Riemannian manifolds with nonnegative Ricci curvature and $p_{j} \in M_{j}$ such that

$$
B_{p_{j}}(1) \stackrel{\mathrm{GH}}{\longrightarrow} B_{0}(1) \subset \mathbb{E}^{m},
$$

where $\mathbb{E}^{m}$ is Euclidean space of dimension $m$. Then

$$
\lim _{j \rightarrow \infty} \operatorname{Vol}\left(B_{p_{j}}(1)\right)=\operatorname{Vol}\left(B_{0}(1)\right) .
$$

Remark 2.22. The proof of this theorem does not require global nonnegative Ricci curvature on a complete manifold. In fact, $M_{j}^{m}$ could be an open manifold as long as $B_{p_{j}}(2) \subset \bar{M}_{j}^{m}$ does not hit the boundary. One may not even need a radius of 2 .

Colding applied this theorem to prove a number of theorems, including one in which the Gromov-Hausdorff limit is an arbitrary compact Riemannian manifold of the same dimension (also [4]):

Theorem 2.23 (Colding). Let $M_{j}^{m}$ and $M_{\infty}^{m}$ be compact Riemannian manifolds with nonnegative Ricci curvature for $j=1,2,3, \ldots$ such that

$$
M_{j}^{m} \stackrel{\mathrm{GH}}{\longrightarrow} M_{\infty}^{m} .
$$

Then for all $r>0$ and for all $p_{j} \in M_{j}$ such that $p_{j} \rightarrow p_{\infty}$, we have

$$
\lim _{j \rightarrow \infty} \operatorname{Vol}\left(B_{p_{j}}(r)\right)=\operatorname{Vol}\left(B_{p_{\infty}}(r)\right) .
$$

Remark 2.24. Again, Colding's proof does not really require $M_{j}$ to be complete. These $M_{j}$ could be open Riemannian manifolds as long as $B_{p_{j}}(r) \subset \bar{M}_{j}$ does not hit the boundary. Here we do not need to worry about twice the radius because the proof involves estimating countable collections of small balls $B_{q_{j, i}}\left(\epsilon_{j, i}\right)$ in $B_{p_{j}}(r)$ and applying Theorem 2.21 to those small balls, and one can always ensure the $B_{q_{j, i}}\left(2 \epsilon_{j, i}\right)$ avoid the boundary as in Remark 2.22. 
Cheeger and Colding then conducted a study of the properties of GromovHausdorff limits of manifolds of nonnegative Ricci curvature in [3]. They improve upon Theorem 2.23, allowing $M_{\infty}$ to be an arbitrary limit space as long as the sequence is noncollapsing:

Theorem 2.25 (Cheeger and Colding). Let $V_{0}>0$ and let $M_{j}^{m}$ be compact Riemannian manifolds with nonnegative Ricci curvature for $j=1,2,3, \ldots$, such that

$$
M_{j}^{m} \stackrel{\mathrm{GH}}{\longrightarrow} M_{\infty}^{m} \text { and } \operatorname{Vol}\left(M_{j}^{m}\right) \geq V_{0} .
$$

Then for all $r>0$ and for all $p_{j} \in M_{j}$ such that $p_{j} \rightarrow p_{\infty} \in M_{\infty}$, we have

$$
\lim _{j \rightarrow \infty} \operatorname{Vol}\left(B_{p_{j}}(r)\right)=\mathscr{H}^{m}\left(B_{p_{\infty}}(r)\right),
$$

where $\mathscr{H}^{m}$ is the Hausdorff measure of dimension $\mathrm{m}$.

Remark 2.26. Again this theorem is proved locally, so as in Remark 2.24 this theorem holds when $M_{j}^{m}$ are open Riemannian manifolds as long as $B_{p_{j}}(r) \subset \bar{M}_{j}^{m}$ do not touch the boundary.

Of course, Cheeger and Colding studied more than just manifolds with nonnegative Ricci curvature and more than just noncollapsing sequences in their work, but these theorems are the only ones needed in this paper. See also work of the second author with Wei for an adaption of their volume convergence theorem which deals with Hausdorff measures defined using restricted versus intrinsic distances [13].

\section{Properties of inner regions}

We defined in Definition 1.3 the inner regions $M^{\delta}$ of an open Riemannian manifold $M$. These spaces are open Riemannian manifolds; however, we will study them using the restricted distance $d_{M}$ rather than the intrinsic length metric $d_{M^{\delta}}$ defined in (4). There are natural isometric embeddings of $\left(M^{\delta}, d_{M}\right)$ and its metric completion $\left(\bar{M}^{\delta}, d_{M}\right)$ into $\left(M, d_{M}\right)$. Thus the metric completion is, in fact, compact when $M$ is precompact. This occurs, for example, when $M$ has finite diameter.

Example 3.1. In Figure 4, we depict a single flat manifold $M^{2}$, which is a flat disk with a spike attached. For a sequence $\delta_{1}<\delta_{2}<\delta_{3}<\delta_{4}$, the gray inner regions depict $M^{\delta_{i}}$. For $\delta$ sufficiently large, $M^{\delta}$ is an empty set.

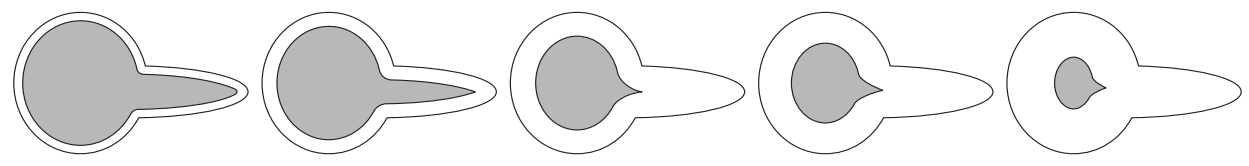

Figure 4. Example 3.1: Single $M$, varying $\delta$. 
Lemma 3.2. For any sequence $\delta_{i} \rightarrow 0$, we have

$$
M=\bigcup_{i=1}^{\infty} M^{\delta_{i}}, \quad \text { and in fact } \quad M=\bigcup_{\delta>0} M^{\delta} .
$$

Proof. Let $x \in M$. Since $M$ is open, $\varepsilon=d_{M}(x, \partial M)>0$. Then $x \in M^{\varepsilon / 2}$.

Lemma 3.3. Let $\delta>\delta^{\prime}>0$. If $y \in M^{\delta}$, then for any $\varepsilon<\delta-\delta^{\prime}$ we have

$$
B(y, \varepsilon)=\left\{x \in M: d_{M}(x, y)<\varepsilon\right\} \subset M^{\delta^{\prime}} .
$$

Proof. Let $x \in B(y, \varepsilon)$, so $d_{M}(x, y)<\delta-\delta^{\prime}$. Since $y \in M^{\delta}$, for all $z \in \partial M$ we have $d_{M}(y, z)>\delta$. By the triangle inequality,

$$
d_{M}(x, z) \geq d_{M}(y, z)-d_{M}(x, y)>\delta-\left(\delta-\delta^{\prime}\right)=\delta^{\prime} .
$$

Inner regions $M^{\delta}$ with restricted metrics $d_{M}$ are not necessarily length spaces:

Example 3.4. In the flat open manifold

$$
M=\left\{(x, y): x^{2}+y^{2} \in(1,25)\right\} \subset \mathbb{E}^{2},
$$

the distance between $(3,1)$ and $(-3,1)$ is

$$
d_{M}((3,1),(-3,1))=6
$$

because they are joined by curves of length arbitrarily close to 6 . However, for $\delta=1$ we have

$$
M^{\delta}=\left\{(x, y): x^{2}+y^{2} \in(4,16)\right\} \subset \mathbb{E}^{2} .
$$

The length of any curve in $M^{\delta}$ between $(3,1)$ and $(-3,1)$ must go around $(0,2)$ and thus has length at least $2 \sqrt{9+1}>6$.

In fact, inner regions of path connected manifolds need not be connected:

Example 3.5. Let $M$ be the connected union of balls in the Euclidean plane:

$$
M=B_{(4,0)}(5) \cup B_{(-4,0)}(5) \subset \mathbb{E}^{2} .
$$

Then

$$
\partial M=A_{+} \cup A_{-},
$$

where

$$
\begin{gathered}
A_{+}=\partial B_{(4,0)}(5) \cap\{(x, y): x \geq 0\}, \\
A_{-}=\partial B_{(-4,0)}(5) \cap\{(x, y):-x \geq 0\} .
\end{gathered}
$$

Note that

$$
(0,3),(0,-3) \in \partial M \text {. }
$$

Thus, for $\delta>3$,

$$
M^{\delta} \cap\{(0, y): y \in \mathbb{R}\}=\varnothing .
$$


However, for $\delta<5$, we have

$$
(4,0),(-4,0) \in M^{\delta} \text {. }
$$

Thus $M^{\delta}$ is not connected for $\delta \in(3,5)$.

\section{Manifolds with Gromov-Hausdorff limits have converging inner regions}

In this section we will prove:

Theorem 4.1. Let $M_{j}$ be precompact open metric spaces, $\left(X, d_{X}\right)$ a compact metric space, and assume $\left(\bar{M}_{j}, d_{M_{j}}\right) \stackrel{\mathrm{GH}}{\longrightarrow}\left(X, d_{X}\right)$. For each $\delta>0$, there exists a sequence of indices $\left\{j_{k}\right\} \rightarrow \infty$ and a compact metric space $Y_{\left\{j_{k}\right\}}^{\delta} \subset X$ such that the subsequence of $\delta$-inner regions $\bar{M}_{j_{k}}^{\delta}$ converges to $Y_{\left\{j_{k}\right\}}^{\delta}$ :

$$
\left(\bar{M}_{j_{k}}^{\delta}, d_{M_{j_{k}}}\right) \stackrel{\mathrm{GH}}{\longrightarrow}\left(Y_{\left\{j_{k}\right\}}^{\delta}, d_{\delta}\right) .
$$

If (17) holds for $\delta=\delta_{1}$ and $\delta=\delta_{2}$, with $0<\delta_{2}<\delta_{1}$, then

$$
Y_{\left\{j_{k}\right\}}^{\delta_{1}} \subset Y_{\left\{j_{k}\right\}}^{\delta_{2}} .
$$

Given a sequence of decreasing positive numbers $\delta_{i} \rightarrow 0$, one can choose the sequence $\left\{j_{k}\right\} \rightarrow \infty$ so that (17) holds for all $\delta=\delta_{i}$; moreover, the union

$$
U_{\left\{\delta_{i}\right\},\left\{j_{k}\right\}}=\bigcup_{i} Y_{\left\{j_{k}\right\}}^{\delta_{i}}
$$

is an open subset of $X$.

Given two sequences $\left\{\delta_{i}\right\},\left\{\beta_{i}\right\}$ such that (17) holds for all $\delta \in\left\{\delta_{i}\right\} \cup\left\{\beta_{i}\right\}$, then

$$
U_{\left\{\delta_{i}\right\},\left\{j_{k}\right\}}=U_{\left\{\beta_{i}\right\},\left\{j_{k}\right\}} .
$$

Note that $M_{j}^{\delta}$ can be an empty space; see Example 4.8. Consider the GromovHausdorff limit of an empty metric space to be an empty metric space.

Remark 4.2. In Example 4.9 we will see that a subsequence $j_{k}$ may be necessary to obtain GH convergence of the $\delta$-inner regions, and that $U_{\left\{\delta_{i}\right\},\left\{j_{k}\right\}}$ depends on the choice of the subsequence. Even the closure of $U_{\left\{\delta_{i}\right\},\left\{j_{k}\right\}}$ may depend on the choice of subsequence $j_{k}$; see Example 4.11. The $U_{\left\{\delta_{i}\right\},\left\{j_{k}\right\}}$ may be disjoint and not isometric; see Example 4.12.

4A. Hausdorff convergence of $\delta$-inner regions. We begin with a basic theorem:

Theorem 4.3. Let $\left(Z, d_{Z}\right)$ be a compact metric space. Suppose $M_{j} \subset Z$ are open metric spaces with the induced metric and $X \subset Z$ is closed and such that $\bar{M}_{j} \stackrel{\mathrm{H}}{\longrightarrow} X$. Then, for each $\delta>0$, there exist a sequence of indices $\left\{j_{k}\right\} \rightarrow \infty$ and a compact set $W_{\left\{j_{k}\right\}}^{\delta} \subset X$ such that

$$
\bar{M}_{j_{k}}^{\delta} \stackrel{\mathrm{H}}{\longrightarrow} W_{\left\{j_{k}\right\}}^{\delta}
$$


If (19) holds for $\delta=\delta_{1}, \delta_{2}$, with $0<\delta_{2}<\delta_{1}$, then

$$
W_{\left\{j_{k}\right\}}^{\delta_{1}} \subset W_{\left\{j_{k}\right\}}^{\delta_{2}} .
$$

Given a sequence of positive numbers $\delta_{i} \rightarrow 0$, there exists a sequence of indices $\left\{j_{k}\right\} \rightarrow \infty$ such that (19) holds for all $\delta=\delta_{i}$; moreover the union

$$
U_{\left\{\delta_{i}\right\},\left\{j_{k}\right\}}^{\prime}=\bigcup_{i} W_{\left\{j_{k}\right\}}^{\delta_{i}}
$$

is an open subset of $X$.

Given two sequences $\left\{\delta_{i}\right\},\left\{\beta_{i}\right\}$ such that (19) holds for all $\delta \in\left\{\delta_{i}\right\} \cup\left\{\beta_{i}\right\}$, we have

$$
U_{\left\{\delta_{i}\right\},\left\{j_{k}\right\}}^{\prime}=U_{\left\{\beta_{i}\right\},\left\{j_{k}\right\}}^{\prime} .
$$

Again here, $M_{j}^{\delta}$ can be an empty space. We consider the Hausdorff limit of an empty metric space to be an empty metric space.

Before we prove this theorem, we provide an example demonstrating that even if

$$
\bar{M}_{j_{k}}^{\delta_{1}} \stackrel{\mathrm{H}}{\longrightarrow} W_{\left\{j_{k}\right\}}^{\delta_{1}} \quad \text { and } \quad \bar{M}_{j_{k}}^{\delta_{2}} \stackrel{\mathrm{H}}{\longrightarrow} W_{\left\{j_{k}\right\}}^{\delta_{2}}
$$

for some $\delta_{1}>\delta_{2}>0$, there may be $\delta \in\left(\delta_{2}, \delta_{1}\right)$ for which $\bar{M}_{j_{k}}^{\delta}$ does not converge: Example 4.4. Fix $\varepsilon<\frac{1}{3}$. In 2-dimensional Euclidean space $\mathbb{E}^{2}$, consider the sequence $M_{j}$, where $M_{2 j}$ is a ball of radius 1 with a spike of width $4 \varepsilon$ attached to it, as depicted in Figure 4, and $M_{2 j+1}$ is a ball of radius 1 with a spike whose width decreases from $6 \varepsilon$ to $4 \varepsilon$ as $j \rightarrow \infty$. Then $\bar{M}_{j}^{\varepsilon}$ converges to ball of radius $1-\varepsilon$ with a spike of width $2 \varepsilon$, and $\bar{M}_{j}^{3 \varepsilon}$ converges to a ball of radius $1-3 \varepsilon$ with no spike attached. But $\bar{M}_{2 j}^{2 \varepsilon}$ converges to a ball of radius $1-2 \varepsilon$, while $\bar{M}_{2 j+1}^{2 \varepsilon}$ converges to a ball of radius $1-2 \varepsilon$ with a line segment attached to it. Thus $M_{j}^{2 \varepsilon}$ does not converge in the Hausdorff sense.

In the proof of Theorem 4.3 we will apply the following fact:

Remark 4.5. Recall that if $\left\{A_{j}\right\}$ is a sequence of closed subsets of a metric space $A$ such that $A_{j} \stackrel{\mathrm{H}}{\longrightarrow} A_{\infty}$, then

$$
A_{\infty}=\left\{a \in A: \text { for all } j \in \mathbb{N} \text {, there exist } a_{j} \in A_{j} \text { such that } \lim _{j \rightarrow \infty} a_{j}=a\right\} .
$$

Any subsequence $\left\{A_{j_{k}}\right\}$ of $\left\{A_{j}\right\}$ also converges in the Hausdorff sense to $A_{\infty}$. Then

$$
A_{\infty}=\left\{a \in A \text { : for all } k \in \mathbb{N} \text {, there exist } a_{j_{k}} \in A_{j_{k}} \text { such that } \lim _{k \rightarrow \infty} a_{j_{k}}=a\right\} .
$$

Proof of Theorem 4.3. Apply Theorem 2.16 to the sequence $\left\{\bar{M}_{j}^{\delta}\right\}_{j=1}^{\infty}$ to get a subsequence $\left\{\bar{M}_{j_{k}}^{\delta}\right\}_{k=1}^{\infty}$ and a compact set $W_{\left\{j_{k}\right\}}^{\delta}$ such that (19) is satisfied. Since $\bar{M}_{j_{k}}^{\delta} \subset \bar{M}_{j_{k}}$, we have $W_{\left\{j_{k}\right\}}^{\delta} \subset X$. Similarly, $W_{\left\{j_{k}\right\}}^{\delta_{1}} \subset W_{\left\{j_{k}\right\}}^{\delta_{2}}$ when (19) holds for $0<\delta_{2}<\delta_{1}$. 
Given $\delta_{i} \rightarrow 0$, start with $\delta_{1}$. By Theorem 2.16 there exists a sequence of integers $\left\{\iota_{k}\left(\delta_{1}\right)\right\} \rightarrow \infty$ and a compact set $W_{\left\{\iota_{k}\left(\delta_{1}\right)\right\}}^{\delta_{1}}$ such that

$$
\bar{M}_{\iota_{k}\left(\delta_{1}\right)}^{\delta_{1}} \stackrel{\mathrm{H}}{\longrightarrow} W_{\left\{\iota_{k}\left(\delta_{1}\right)\right\}}^{\delta_{1}} \cdot
$$

For $n>1$, there exists a subsequence $\left\{\iota_{k}\left(\delta_{n}\right)\right\}_{k}$ of $\left\{\iota_{k}\left(\delta_{n-1}\right)\right\}_{k}$ and a compact set $W_{\left\{\iota_{k}\left(\delta_{n}\right)\right\}}^{\delta_{n}}$ such that

$$
\bar{M}_{\iota_{k}\left(\delta_{n}\right)}^{\delta_{n}} \stackrel{\mathrm{H}}{\longrightarrow} W_{\left\{\iota_{k}\left(\delta_{n}\right)\right\}}^{\delta_{n}} \cdot
$$

Define $j_{k}=\iota_{k}\left(\delta_{k}\right)$. Then $\left\{j_{k}\right\}_{k=n}^{\infty}$ is a subsequence of $\left\{\iota_{k}\left(\delta_{n}\right)\right\}_{k=1}^{\infty}$, and thus (19) holds for all $n$.

Let $y$ be an element of $U_{\left\{\delta_{i}\right\},\left\{j_{k}\right\}}^{\prime}$. There exists $N \in \mathbb{N}$ such that $y \in W_{\left\{J_{k}\right\}}^{\delta_{i}}$ for $i \geq N$. Suppose that $x \in X$ and $d_{Z}(x, y)<\delta_{N} / 6$. Since $y \in W_{\left\{j_{k}\right\}}^{\delta_{i}}$, choose $y_{j_{k}} \in \bar{M}_{j_{k}}^{\delta_{N}}$ such that $y=\lim _{j \rightarrow \infty} y_{j_{k}}$ and $d_{Z}\left(y, y_{j_{k}}\right)<\delta_{N} / 6$. Analogously, take $x_{j} \in \bar{M}_{j}$ such that $x=\lim _{j \rightarrow \infty} x_{j}$ and $d_{Z}\left(x, x_{j}\right)<\delta_{N} / 6$. Then

$$
d_{Z}\left(x_{j_{k}}, y_{j_{k}}\right)<d_{Z}\left(x_{j_{k}}, x\right)+d_{Z}(x, y)+d_{Z}\left(y, y_{j_{k}}\right)<\frac{\delta_{N}}{2} .
$$

This implies that $d_{Z}\left(x_{j_{k}}, \partial\left(M_{j_{k}}\right)\right)>\delta_{N} / 2$. Then $x \in W_{\left\{J_{k}\right\}}^{\delta_{i}} \subset U_{\left\{\delta_{i}\right\},\left\{j_{k}\right\}}^{\prime}$ for some $i>N$.

Given another sequence $\beta_{i} \rightarrow 0$ such that (19) holds for all $\delta=\beta_{i}$, select for each $i$ some $l(i)$ such that $\delta_{l(i)}<\beta_{i}$. Then

$$
W_{\left\{j_{k}\right\}}^{\beta_{i}} \subset W_{\left\{j_{k}\right\}}^{\delta_{l(j)}}
$$

and so $U_{\left\{\beta_{i}\right\},\left\{j_{k}\right\}}^{\prime} \subset U_{\left\{\delta_{l(i)}\right\},\left\{j_{k}\right\}}^{\prime} \subset U_{\left\{\delta_{i}\right\},\left\{j_{k}\right\}}^{\prime}$. Conversely, $U_{\left\{\delta_{i}\right\},\left\{j_{k}\right\}}^{\prime} \subset U_{\left\{\beta_{i}\right\},\left\{j_{k}\right\}}^{\prime}$.

Definition 4.6. With the hypotheses of Theorem 4.3, define

$$
U_{\left\{j_{k}\right\}}^{\prime}=\bigcup W_{\left\{j_{k}\right\}}^{\delta},
$$

where the union is taken over all $\delta$ for which $\bar{M}_{j_{k}}^{\delta}$ is a sequence that converges in the Hausdorff sense to a metric space $W_{\left\{j_{k}\right\}}^{\delta}$, and define

$$
U^{\prime}=\bigcup_{\delta>0} W^{\delta}
$$

where $W^{\delta}$ is the Hausdorff limit space of some convergent subsequence of $\bar{M}_{j}^{\delta}$.

\section{B. Finding limits of inner regions in the Gromov-Hausdorff limits.}

Proof of Theorem 4.1. By Theorem 2.15 there exists a common metric space $Z$ and isometric embeddings $\varphi_{j}:\left(\bar{M}_{j}, d_{M_{j}}\right) \rightarrow\left(Z, d_{Z}\right), \varphi:\left(X, d_{X}\right) \rightarrow\left(Z, d_{Z}\right)$ such that

$$
d_{H}^{Z}\left(\varphi_{j}\left(\bar{M}_{j}\right), \varphi(X)\right) \rightarrow 0 .
$$


Now we can apply Theorem 4.3. For each $\delta>0$, there exist a subsequence $\varphi_{j_{k}}\left(\bar{M}_{j_{k}}^{\delta}\right)$ and a compact set $W_{\left\{j_{k}\right\}}^{\delta} \subset \varphi(X)$ such that

$$
\varphi_{j_{k}}\left(\bar{M}_{j_{k}}^{\delta}\right) \stackrel{\mathrm{H}}{\longrightarrow} W_{\left\{j_{k}\right\}}^{\delta} \text {. }
$$

Let $Y_{\left\{j_{k}\right\}}^{\delta}=\varphi^{-1}\left(W_{\left\{j_{k}\right\}}^{\delta}\right)$. Clearly, (17) holds and $Y_{\left\{j_{k}\right\}}^{\delta_{1}} \subset Y_{\left\{j_{k}\right\}}^{\delta_{2}}$ when (17) holds for $0<\delta_{2}<\delta_{1}$. Given a sequence of positive numbers $\delta_{i} \rightarrow 0$, there exists a subsequence $\left\{j_{k}\right\} \subset \mathbb{N}$ such that

$$
\varphi_{j_{k}}\left(\bar{M}_{j_{k}}^{\delta_{i}}\right) \stackrel{\mathrm{H}}{\longrightarrow} W_{\left\{j_{k}\right\}}^{\delta_{i}}
$$

for all $i$. Then (17) holds for all $i$, and $U_{\left\{\delta_{i}\right\},\left\{j_{k}\right\}}=\varphi^{-1}\left(U_{\left\{\delta_{i}\right\},\left\{j_{k}\right\}}^{\prime}\right)$ is an open subset of $X$ that does not depend on the sequence $\delta_{i}$.

4C. Unions of limits of inner regions in Gromov-Hausdorff limits. The following notion of an "inner union" has some interesting properties.

Definition 4.7. In the situation of Theorem 4.1, let $D_{\left\{j_{k}\right\}}$ denote the set of $\delta>0$ such that (17) holds for a given sequence $\left\{j_{k}\right\}$. We put

$$
U_{\left\{j_{k}\right\}}=\bigcup_{\delta \in D_{\left\{j_{k}\right\}}} Y_{\left\{j_{k}\right\}}^{\delta}
$$

and call this set an inner union of limits for the sequence $\left\{M_{j}\right\}$. Observe that, by (18), we have

$$
U_{\left\{j_{k}\right\}}=U_{\left\{\delta_{i}\right\},\left\{j_{k}\right\}}
$$

for any sequence $\left\{\delta_{i}\right\} \rightarrow 0$ of elements of $D_{\left\{j_{k}\right\}}$.

In Theorem 6.6 we will prove that $U_{\left\{j_{k}\right\}}$ is a special case of the glued limits we will construct in Theorem 6.3. Since it is easy to understand the properties of these $U_{\left\{j_{k}\right\}}$, we present a few examples of them here so that we may refer to them later as examples of glued limit spaces.

Example 4.8. Let $M_{j}$ be a Euclidean disk of radius $1 / j$. Then $\bar{M}_{j} \stackrel{\mathrm{GH}}{\longrightarrow} X$, where $X$ is a single point. For any $\delta>0$, taking $j>1 / \delta$, we have $M_{j}^{\delta}=\varnothing$. Thus the inner union of limits is empty for any choice of subsequence.

In the following example we see that $U_{\left\{j_{k}\right\}}$ depends on the subsequence $\left\{j_{k}\right\}$, and in the next we see that $X$ is not necessarily contained in the closure of $U_{\left\{j_{k}\right\}}$, even if the closure is nonempty.

Example 4.9. Let $M_{2 j}$ be the Euclidean disk of radius 1 and $M_{2 j+1}$ the Euclidean disk with the center point removed. Then $\bar{M}_{j}$ is a closed Euclidean disk as is the limit space $X$. Given $\delta \in(0,1), M_{2 j}^{\delta}$ is the Euclidean disk of radius $1-\delta$. Their metric completions converge to the closed disk of radius $1-\delta . U_{\{2 j\}}$ is the open Euclidean disk of radius 1 . However, $M_{2 j+1}^{\delta}$ is a Euclidean annulus $\operatorname{Ann}_{0}(\delta, 1-\delta)$, 
and the metric completions converge to the closure of this annulus. $U_{\{2 j+1\}}$ is the open Euclidean disk of radius 1 with the center point removed. In this example $U=U_{\{2 j\}}$.

Example 4.10. In 2-dimensional Euclidean space, consider the sequence of balls with attached spikes depicted in Figure 4. The Gromov-Hausdorff limit of the sequence is a ball with an interval attached, while the closure of $U$ is just the closed ball.

In Example 4.9, we saw that $U_{\{2 j\}} \neq U_{\{2 j+1\}}$, yet their closures are the same. This is not always the case; $U_{\left\{j_{k}\right\}}$ could even be an empty set.

Example 4.11. For $j \in \mathbb{N}$, let $M_{2 j}$ be a flat torus (so it has no boundary), and let the $M_{2 j+1}$ be flat tori with increasingly dense small holes cut out, the holes getting smaller and smaller so the $M_{2 j+1}$ still converge to the flat torus $X$. Then $U_{\{2 j\}}=X$, but for any $\delta>0, M_{2 j+1}^{\delta}$ becomes an empty set. So $U_{\{2 j+1\}}$ is the empty set.

Example 4.12. For $j \in \mathbb{N}$, let $M_{2 j}$ be a flat torus $S^{1} \times S^{1}$, with increasingly many dense small holes in $W \times S^{1}$, where $W=(0, \pi / 4) \subset S^{1}$, and let $M_{2 j+1}$ be a flat torus $S^{1} \times S^{1}$, with increasingly many dense small holes in $\left(S^{1} \backslash W\right) \times S^{1}$. Then

$$
U_{\{2 j\}}=\left(S^{1} \backslash W\right) \times S^{1} \quad \text { and } \quad U_{\{2 j+1\}}=W \times S^{1}
$$

with the restricted distance from $S^{1} \times S^{1}$, which are disjoint and not isometric to each other.

Example 4.13. It is possible for a sequence of open Riemannian manifolds $M_{j}$ to have $\delta$-inner regions $M_{j}^{\delta}$ which converge in the Gromov-Hausdorff sense to some $Y^{\delta}$ for all $\delta>0$, and yet the limit has two distinct inner unions $U_{\{2 j\}} \neq$ $U_{\{2 j+1\}}$. This can be seen, for example, with the following F-shaped regions:
$M_{j}=(0,1 / j) \times(-1,0]$
$(0,1) \times(0,3)$
$[1,3) \times(0,1)$
$[1,3) \times(2,3) \backslash A_{j}$

in the Euclidean plane, where $A_{2 j}$ is an increasingly dense collection of increasingly tiny balls in $(1,3) \times(0,1)$, and $A_{2 j+1}$ is an increasingly dense collection of increasingly tiny balls in $(1,3) \times(2,3)$. Then

$$
M_{j} \stackrel{\mathrm{GH}}{\longrightarrow} X=(0,1) \times(0,3) \cup[1,3) \times(0,1) \cup[1,3) \times(2,3) .
$$

For $\delta>0$ fixed, taking $j$ large enough that $1 / j<2 \delta$, we see that

$$
M_{2 j}^{\delta} \stackrel{\mathrm{GH}}{\longrightarrow} Y^{\delta}=(\delta, 1-\delta) \times(\delta, 3-\delta) \cup[1-\delta, 3-\delta) \times(2+\delta, 3-\delta),
$$

which is isometric to

$$
M_{2 j+1}^{\delta} \stackrel{\mathrm{GH}}{\longrightarrow} Y^{\delta}=(\delta, 1-\delta) \times(\delta, 3-\delta) \cup[1-\delta, 3-\delta) \times(\delta, 1-\delta) .
$$


Thus, the $M_{j}^{\delta}$ have a GH limit without taking a subsequence. On the other hand, the inner unions of limits are not equal, only isometric:

$$
\begin{aligned}
U_{\{2 j\}} & =(0,1) \times(0,3) \cup[1,3) \times(2,3) \subset X, \\
U_{\{2 j+1\}} & =(0,1) \times(0,3) \cup[1,3) \times(0,1) \subset X .
\end{aligned}
$$

We will prove in Theorem 6.6 that when $M_{j}^{\delta}$ have GH limits for all $\delta$, all closures of inner unions of limits are isometric.

\section{Converging inner regions of sequences with curvature bounds}

In this section, we prove that $\delta$-inner regions converge under certain geometric hypotheses on the manifolds even when the manifolds themselves have no GromovHausdorff limits.

5A. Constant sectional curvature. Here we prove that the inner regions of a sequence of manifolds in the following class have a subsequence which converges in the Gromov-Hausdorff sense.

Definition 5.1. Given $m \in \mathbb{N}, H \in \mathbb{R}, V>0$, and $l>0$, we define $\mu_{H}^{m, V, l}$ to be the class of connected open Riemannian manifolds $M$ of dimension at most $m$ with constant sectional curvature $\operatorname{Sect}_{M}=H, \operatorname{Vol}(M) \leq V$, and

$$
L_{\min }(M)=\inf \left\{L_{g}(C): C \text { is a closed geodesic in } \mathrm{M}\right\}>l,
$$

where a closed geodesic is any geodesic which starts and ends at the same point.

Recall that complete simply connected manifolds with constant sectional curvature $H \leq 0$ have no closed geodesics, by Hadamard's theorem, while those with $H>0$ have $L(M)=2 \pi / \sqrt{H}$. (See [6].) Here we are requiring that the closed geodesic lies in an open manifold $M$, and we do not have completeness.

Theorem 5.2. Given any $\delta>0$, if $\left(M_{j}, g_{j}\right) \subset M_{H}^{m, V, l}$, then there is a subsequence $\left(M_{j_{k}}^{\delta}, d_{M_{j_{k}}}\right)$ such that the metric completion with the restricted metric converges in the Gromov-Hausdorff sense to a metric space $\left(Y^{\delta}, d\right)$. In particular, the extrinsic diameters measured using the restricted metric are bounded uniformly:

where

$$
\operatorname{Diam}\left(M_{j_{k}}^{\delta}, d_{M_{j_{k}}}\right) \leq \epsilon_{0} \frac{V}{V_{H}^{m}\left(\epsilon_{0}\right)}, \quad \operatorname{Diam}\left(Y^{\delta}, d\right) \leq \epsilon_{0} \frac{V}{V_{H}^{m}\left(\epsilon_{0}\right)},
$$

$$
\epsilon_{0}= \begin{cases}\frac{1}{2} \min \left\{\delta, \frac{l}{2}, \frac{\pi}{\sqrt{H}}\right\} & \text { if } H>0, \\ \frac{1}{2} \min \left\{\delta, \frac{l}{2}\right\} & \text { otherwise, }\end{cases}
$$

and $V_{H}^{m}\left(\epsilon_{0}\right)$ is the volume of a ball of radius $\epsilon_{0}$ in the complete simply connected space with constant sectional curvature $H$. 
Remark 5.3. There are no closed geodesics in the $M_{j}$ of Examples 1.1 and 1.2, so $L\left(M_{j}\right)=\infty$. These examples have $H=0$ and $m=2$. Since Example 1.2 also has a uniform upper bound on volume, it demonstrates why we can only obtain Gromov-Hausdorff convergence of the $M_{j}^{\delta}$ instead of the $M_{j}$ themselves. The $M_{j}^{\delta}$ of Example 1.1 do not have GH-convergent subsequences (see Remark 5.5), demonstrating the necessity of an upper bound on volume.

Proof of Theorem 5.2. Let $M \in M_{H}^{m, V, l}$ and $p \in M^{\delta}$. In view of (26), we see that if $0<\epsilon<\epsilon_{0}$, then $B_{p}(\epsilon)$ does not reach the boundary of $M$ and does not contain any conjugate point to $p$, since one does not reach a conjugate point before one would in the comparison space.

We claim that there are also no cut points to $p$ in $B_{p}(\epsilon)$. If there was a cut point $q$, then proceeding in a similar way to Klingenberg [9], we see that there exists a closed geodesic starting at $p$ of length at most $2 d(p, q)<2 \epsilon_{0}$. By hypothesis, the length of this closed geodesic is greater than $l$, which is a contradiction.

Thus there is a Riemannian isometric diffeomorphism

$$
\psi: B_{p}\left(\epsilon_{0}\right) \rightarrow B_{x}\left(\epsilon_{0}\right) \subset M_{H}^{m}
$$

where $M_{H}^{m}$ is the simply connected space of constant sectional curvature $H$. In particular, $\operatorname{Vol}\left(B_{p}(\epsilon)\right)$ is greater than or equal to the volume of a ball of the same radius in a simply connected space form of constant curvature $H$. By combining Proposition 2.11 with Proposition 2.10 and then Gromov's compactness theorem, there is a subsequence $\left(M_{j_{k}}^{\delta}, d_{M_{j_{k}}}\right)$ such that the metric completion with the restricted metric converges in the Gromov-Hausdorff sense to a metric space $\left(Y^{\delta}, d\right)$. Notice that by Proposition 2.11, the maximum number of disjoint balls of radius $\epsilon_{0} / 2$ that lie in $M$ is at most $\left(V / V_{H}^{m}\right)\left(\epsilon_{0} / 2\right)$. Thus, by Proposition 2.10, the minimum number of balls of radius $\epsilon_{0}$ needed to cover $M$ is at most $\left(V / V_{H}^{m}\right)\left(\epsilon_{0} / 2\right)$. From this it follows that

$$
\operatorname{Diam}\left(M_{j_{k}}^{\delta}, d_{M_{j_{k}}}\right) \leq \epsilon_{0} \frac{V}{V_{H}^{m}\left(\epsilon_{0} / 2\right)}
$$

Since

$$
\operatorname{Diam}\left(M_{j_{k}}^{\delta}, d_{M_{j_{k}}}\right) \rightarrow \operatorname{Diam}\left(Y^{\delta}, d\right)
$$

we conclude that

$$
\operatorname{Diam}\left(Y^{\delta}, d\right) \leq \epsilon_{0} \frac{V}{V_{H}^{m}\left(\epsilon_{0} / 2\right)}
$$

Remark 5.4. If the injectivity radius for each $p \in M_{j}^{\delta}$ is bounded above by a positive constant, then the condition on the length of closed geodesics in Theorem 5.2 is satisfied. 
5B. Examples with constant sectional curvature. The volume condition in Theorem 5.2 may not be replaced by a condition on diameter:

Remark 5.5. Let $\left(M_{j}, g_{j}\right)$ be the $j$-th covering space of $\operatorname{Ann}_{(0,0)}(1 / j, 1) \subset \mathbb{E}^{2}$.

Since every point in $M_{j}$ has distance less than 1 from the inner boundary, and the inner boundary has length $j 2 \pi(1 / j)=2 \pi$, we know

$$
\operatorname{Diam}\left(M_{j}, d_{M_{j}}\right) \leq 2 \pi+2 .
$$

Yet the number of disjoint balls of radius $\delta<\frac{1}{4}$ centered on the cover of $\partial B_{(0,0)}\left(\frac{1}{2}\right)$ is greater than $2 j$. So there is no subsequence of $M_{j}^{\delta}$ which converges in the Gromov-Hausdorff sense.

This sequence fails to satisfy the volume condition of Theorem 5.2:

$$
\operatorname{Vol}\left(M_{j}\right)=j\left(\pi 1^{2}-\pi / j^{2}\right)=\pi \frac{j^{2}-1}{j} .
$$

It is worth observing that the intrinsic diameters

$$
\operatorname{Diam}\left(M_{j}^{\delta}, M_{j}^{\delta}\right) \geq j 2 \pi\left(\delta+\frac{1}{j}\right)
$$

also diverge to infinity.

Remark 5.6. The flat manifolds of Example 1.2, described more explicitly in Example 2.13, satisfy the hypothesis of Theorem 5.2. See Figure 5. In fact, for fixed $\delta>0$, once $(2 \pi / j) 4<\delta$, every point with $r \geq 2$ lies within a distance $\delta$ from the boundary because the spike is less than $\delta$ wide. So all the $M_{j}^{\delta}$ eventually lie within $r<2$, where the metric is just the standard Euclidean metric, and there is a uniform bound on the number of disjoint balls. So the Gromov-Hausdorff limit also lies within the Euclidean ball of radius 2. On the other hand, every point within the ball of radius $1+\delta<r<2-\delta$ lies in $M_{j}^{\delta}$, so the Gromov-Hausdorff limit $Y^{\delta}$ contains $\operatorname{Ann}_{(0,0)}(1+\delta, 2-\delta)$. In fact, $Y^{\delta}$ is the metric completion of this annulus with the flat Euclidean metric.

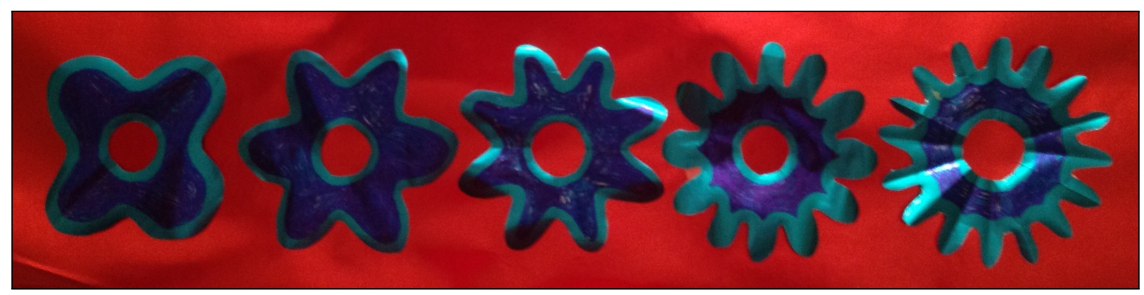

Figure 5. Models of Example 1.2: $M_{4}^{\delta}, M_{6}^{\delta}, M_{8}^{\delta}, M_{12}^{\delta}, M_{16}^{\delta}, \ldots$ 
5C. Manifolds with nonnegative Ricci curvature. Here we prove Theorem 1.4 by applying Gromov's compactness theorem (Theorem 2.9) combined with the following proposition:

Proposition 5.7. If $\left(M, g_{M}\right)$ is a compact Riemannian manifold with boundary having nonnegative Ricci curvature, then for any $\delta>0$ and any $\epsilon \in(0, \delta / 2)$, the $\delta$-inner region $M^{\delta}$ contains a finite collection of points $\left\{p_{1}, p_{2}, \ldots, p_{N}\right\}$ such that

where

$$
M^{\delta} \subset \bigcup_{i=1}^{N} B_{p_{i}}(\epsilon),
$$

$$
N \leq N\left(\delta, \epsilon, D_{\delta}, V, \theta\right)=\frac{V}{\theta}\left(\frac{2^{2 D_{\delta} / \epsilon}}{\epsilon}\right)^{m},
$$

$m=\operatorname{dim}(M), \operatorname{Vol}(M) \leq V, \operatorname{Diam}\left(M^{\delta}, d_{M^{\delta}}\right) \leq D_{\delta}$, and

$$
\sup \left\{\operatorname{Vol}\left(B_{q}(\delta)\right): q \in M^{\delta}\right\} \geq \theta \delta^{m} .
$$

Remark 5.8. In this proposition we can use the volume of any ball centered in $M^{\delta}$ to estimate $\theta$ in (29). This allows us to study sequences like those in Example 3.1. One does not need a Ricci curvature condition if one has a uniform lower bound on the volumes of all balls centered in $M^{\delta}$, as can be seen in Proposition 2.11 in the review of Gromov-Hausdorff convergence.

Proof of Proposition 5.7. By Propositions 2.10 and 2.11 in the review of GromovHausdorff convergence, we need only to find a uniform lower bound on the volume of an arbitrary ball $B_{p}(\epsilon)$ centered at $p \in M^{\delta}$.

Fix $q$ as in (6). Then by the fact that $B_{q}(\delta)$ does not hit $\partial M$ and $M$ has nonnegative Ricci curvature, we may apply the Bishop-Gromov volume comparison theorem to see that

$$
\theta \leq \frac{\operatorname{Vol}\left(B_{q}(\delta)\right)}{\delta^{m}} \leq \frac{\operatorname{Vol}\left(B_{q}(\epsilon)\right)}{\epsilon^{m}}
$$

because $\delta>\delta / 2>\epsilon$.

Let $C:[0,1] \rightarrow M^{\delta}$ be the shortest curve from $p$ to $q$. Then

$$
L=L(C) \leq \operatorname{Diam}\left(M^{\delta}, d_{M^{\delta}}\right) \leq D_{\delta} .
$$

Let $n>L / \epsilon$ and $x_{j}=C\left(t_{j}\right)$, where $t_{j}=j L / n$, so that

$$
x_{j} \in M^{\delta} \quad \text { and } \quad d_{M}\left(x_{j-1}, x_{j}\right)=L / n<\epsilon .
$$

In particular, $B_{x_{j}}(2 \epsilon)$ lies within the interior of $M$ and has nonnegative Ricci curvature. Thus, by the Bishop-Gromov volume comparison theorem,

$$
\operatorname{Vol}\left(B_{x_{j}}(\epsilon)\right) \geq \frac{1}{2^{m}} \operatorname{Vol}\left(B_{x_{j}}(2 \epsilon)\right) \geq \frac{1}{2^{m}} \operatorname{Vol}\left(B_{x_{j+1}}(\epsilon)\right) \text {. }
$$


Applying this repeatedly for $j=1, \ldots, n$, and finally applying (30), we have

$$
\operatorname{Vol}\left(B_{p}(\epsilon)\right) \geq \frac{1}{2^{m n}} \operatorname{Vol}\left(B_{q}(\epsilon)\right) \geq \frac{1}{2^{m D_{\delta} / \epsilon}} \operatorname{Vol}\left(B_{q}(\epsilon)\right) \geq \frac{1}{2^{m D_{\delta} / \epsilon}} \theta \epsilon^{m} .
$$

The estimate on $N\left(\delta, \epsilon, D_{\delta}, V, \theta\right)$ then follows immediately from Propositions 2.10 and 2.11 .

\section{Glued limit spaces}

In this section we define glued limit spaces and completed glued limit spaces and study their properties without making any curvature assumptions. We begin by constructing isometric embeddings

$$
\varphi_{\delta_{i+1}, \delta_{i}}: Y^{\delta_{i+1}} \rightarrow Y^{\delta_{i}}
$$

between the Gromov-Hausdorff limits $Y^{\delta_{i}}$ of inner regions $M_{j}^{\delta_{i}}$ (Theorem 6.1). We then apply these isometric embeddings to glue together the $Y^{\delta_{i}}$ and construct a glued limit space $Y=Y\left(\left\{\delta_{i}\right\},\left\{\varphi_{\delta_{i+1}}, \delta_{i}\right\}\right)$ (Theorem 6.3).

We next study sequences of $M_{j}$ which converge in the Gromov-Hausdorff sense. We prove that if the sequence has a completed glued limit space, then it is unique (Theorem 6.6). However, the glued limit is not the Gromov-Hausdorff limit (Remark 6.7), it might even be empty (Remark 6.8), and it need not exist (Remark 6.9).

Finally, we construct some important examples of glued limit spaces for sequences which do not have Gromov-Hausdorff limits. In Remark 6.10 we describe how the sequence from Example 2.13 has a bounded and precompact glued limit space. We provide another example with a bounded glued limit space which is not precompact (Example 6.12). We provide an example where the glued limit space is not a length space (Example 6.13). We close this section with Example 6.16 demonstrating that these glued limit spaces and their completions depend on the isometric embeddings used to define them and need not be unique.

6A. Gluing inner regions together. Here we prove the existence of isometric embeddings which we will later apply as glue to connect inner regions together.

Theorem 6.1. Let $\delta_{i} \rightarrow 0$ be a decreasing sequence and $M_{j}$ a sequence of open manifolds such that

$$
\left(\bar{M}_{j}^{\delta_{i}}, d_{M_{j}}\right) \stackrel{\mathrm{GH}}{\longrightarrow}\left(Y^{\delta_{i}}, d_{Y^{\delta_{i}}}\right)
$$

for all $i$, where possibly some of these sequences and their limits are eventually empty sets. Then there exist subsequential limit isometric embeddings

$$
\varphi_{\delta_{i+1}, \delta_{i}}: Y^{\delta_{i}} \rightarrow Y^{\delta_{i+1}}
$$


which are just the identity when $\delta_{i}=\delta_{i+1}$. If $\delta \in\left(0, \delta_{0}\right]$, there exists a compact metric space $Y^{\delta_{i}} \subset Y^{\delta} \subset Y^{\delta_{i+1}}$ with the restricted metric $d_{Y^{\delta}}=d_{Y^{\delta_{i+1}}}$ and a convergent subsequence

$$
\left(\bar{M}_{j_{k}}^{\delta}, d_{M_{j_{k}}}\right) \stackrel{\mathrm{GH}}{\longrightarrow}\left(Y^{\delta}, d_{Y^{\delta}}\right),
$$

and when $\delta \in\left(\delta_{i+1}, \delta_{i}\right)$, for any such $Y^{\delta}$, the restriction map $\varphi_{\delta, \delta_{i}}: Y^{\delta_{i}} \rightarrow Y^{\delta}$ and the inclusion map $\varphi_{\delta_{i+1}, \delta}: Y^{\delta} \rightarrow Y^{\delta_{i+1}}$ are isometric embeddings.

Proof. By Theorem 2.15, for each $i$ there exists a compact metric space $Z_{i}$ and isometric embeddings

$$
\varphi_{j}: \bar{M}_{j}^{\delta_{i+1}} \rightarrow Z_{i} \quad \text { and } \quad \varphi_{\infty}: Y^{\delta_{i+1}} \rightarrow Z_{i}
$$

such that

$$
\varphi_{j}\left(\bar{M}_{j}^{\delta_{i+1}}\right) \stackrel{\mathrm{H}}{\longrightarrow} \varphi_{\infty}\left(Y^{\delta_{i+1}}\right) .
$$

By Theorem 2.16, we can choose a subsequence $\left\{j_{k}\right\}_{k=1}^{\infty}$ such that the $\varphi_{j_{k}}\left(\bar{M}_{j_{k}}^{\delta_{i}}\right)$ converge in the Hausdorff sense to a compact subspace $X^{\delta_{i}} \subset \varphi_{\infty}\left(Y^{\delta_{i+1}}\right)$. By (31),

$$
\bar{M}_{j_{k}}^{\delta_{i}} \stackrel{\mathrm{GH}}{\longrightarrow} Y^{\delta_{i}} .
$$

Then, by uniqueness, up to an isometry of the Gromov-Hausdorff limit space there exists an isometric embedding

$$
\varphi_{\delta_{i+1}, \delta_{i}}: Y^{\delta_{i}} \rightarrow Y^{\delta_{i+1}} \quad \text { such that } \quad \varphi_{\delta_{i+1}, \delta_{i}}\left(Y^{\delta_{i}}\right)=\varphi_{\infty}^{-1}\left(X^{\delta_{i}}\right) .
$$

By Theorem 2.12 there is a uniform upper bound $D_{i}>0$ of the diameters of $\left(\bar{M}_{j}^{\delta_{i}}, d_{M_{j}}\right)$ and a function $N_{i}:\left(0, D_{i}\right] \rightarrow \mathbb{N}$ such that $N_{i}(\epsilon)$ is an upper bound for the number of $\epsilon$-balls needed to cover $\bar{M}_{j}^{\delta_{i}}$ for all $\epsilon \in\left(0, D_{i}\right]$ and for all $j \in \mathbb{N}$. If $\delta \in\left(\delta_{i+1}, \delta_{i}\right)$, define $N:\left(0, D_{i+1} / 2\right] \rightarrow \mathbb{N}$ by $N(\epsilon)=N_{i+1}(2 \epsilon)$. Then

$$
\operatorname{Diam}\left(\bar{M}_{j}^{\delta}, d_{M_{j}}\right) \leq \operatorname{Diam}\left(\bar{M}_{j}^{\delta_{i+1}}, d_{M_{j}}\right) \leq D_{i+1} .
$$

Apply Theorem 2.9 to get a subsequence $\left\{l_{k}\right\}_{k=1}^{\infty}$ of $\left\{j_{k}\right\}_{k=1}^{\infty}$ such that the $\varphi_{l_{k}}\left(\bar{M}_{l_{k}}^{\delta_{i}}\right)$ converge in the Hausdorff sense to a closed subset $X^{\delta} \subset \varphi_{\infty}\left(Y^{\delta_{i+1}}\right)$.

We define

$$
Y^{\delta}=\varphi_{\infty}^{-1}\left(X_{\delta}\right) \subset Y^{\delta_{i+1}}
$$

The choice of the subsequence $\left\{l_{k}\right\}$ implies that $X^{\delta_{i}} \subset Y^{\delta}$, so $Y^{\delta_{i}} \subset Y^{\delta}$. The rest of the theorem immediately follows.

Remark 6.2. The choice of isometric embeddings $\varphi_{\delta_{i+1}, \delta_{i}}$ is not unique. In Example 6.16 we provide two distinct isometric embeddings $\varphi_{\delta_{i+1}, \delta_{i}} \neq \varphi_{\delta_{i+1}, \delta_{i}}^{\prime}$. 
6B. Glued limit spaces are defined. We now define a glued limit space for a sequence of Riemannian manifolds satisfying the hypothesis of Theorem 6.1. We prove that this glued limit space is a metric space unless it is the empty set. We prove that it contains isometric images of all Gromov-Hausdorff limits of convergent subsequences of inner regions (which may be empty). An example of a sequence of open Riemannian manifolds which has an empty glued limit space will be given in Remark 6.8. Our definitions of a glued limit space and a completed glued limit space are stated along with their construction in the following theorem:

Theorem 6.3. Given a sequence of open Riemannian manifolds $M_{j}$ with a sequence $\delta_{i} \rightarrow 0$ satisfying the hypothesis of Theorem 6.1, one can define a glued limit space $Y$ using the subsequential limit isometric embeddings of (32) as follows:

$$
Y=Y\left(\left\{\delta_{i}\right\},\left\{\varphi_{\delta_{i+1}, \delta_{i}}\right\}\right)=Y^{\delta_{0}} \sqcup \bigsqcup_{i=1}^{\infty}\left(Y^{\delta_{i+1}} \backslash \varphi_{\delta_{i+1}, \delta_{i}}\left(Y^{\delta_{i}}\right)\right)
$$

with the metric

$$
\begin{aligned}
& d_{Y}(x, y)= \\
& \left\{\begin{array}{l}
d_{Y^{\delta_{0}}}(x, y) \\
d_{Y^{\delta_{i+1}}}(x, y) \\
d_{Y^{\delta_{i+1}}}\left(x, \varphi_{\delta_{i+1}, \delta_{0}}(y)\right) \\
d_{Y^{\delta_{i+j+1}}}\left(x, \varphi_{\delta_{i+j+1}, \delta_{i+1}}(y)\right)
\end{array}\right.
\end{aligned}
$$

$$
\begin{aligned}
& \text { if } x, y \in Y^{\delta_{0}}, \\
& \text { if } x, y \in Y^{\delta_{i+1}} \backslash \varphi_{\delta_{i+1}, \delta_{i}}\left(Y^{\delta_{i}}\right) \text {, } \\
& \text { if } x \in Y^{\delta_{i+1}} \backslash \varphi_{\delta_{i+1}, \delta_{i}}\left(Y^{\delta_{i}}\right) \text { for some } i \in \mathbb{N} \\
& \text { and } y \in Y^{\delta_{0}}, \\
& \text { if } x \in Y^{\delta_{i+j+1}} \backslash \varphi_{\delta_{i+j+1}, \delta_{i+j}}\left(Y^{\delta_{i+j}}\right) \text { and } \\
& y \in Y^{\delta_{i+1}} \backslash \varphi_{\delta_{i+1}, \delta_{i}}\left(Y^{\delta_{i}}\right) \text { for some } i, j \in \mathbb{N} \text {, }
\end{aligned}
$$

where we have set

$$
\varphi_{\delta_{i+j}, \delta_{i}}=\varphi_{\delta_{i+j}, \delta_{i+j-1}} \circ \cdots \circ \varphi_{\delta_{i+1}, \delta_{i}} .
$$

This glued limit is not defined using an arbitrary collection of isometric embeddings, but rather only those achieved as in Theorem 6.1.

Furthermore, for all $\delta \in\left(0, \delta_{0}\right]$ there exists a subsequence $M_{j_{k}}^{\delta}$ which converges in the Gromov-Hausdorff sense to a compact metric space $Y^{\delta}$, and for any such $Y^{\delta}$ there exists an isometric embedding

$$
F_{\delta}=F_{\delta,\left\{\delta_{i}\right\}}: Y^{\delta} \rightarrow Y
$$

such that for the $\delta_{i}$ in our sequence we have

$$
F_{\delta_{i}}\left(Y^{\delta_{i}}\right) \subset F_{\delta_{i+1}}\left(Y^{\delta_{i+1}}\right) .
$$

If $\beta_{j}$ is any sequence decreasing to 0 , then

$$
Y=\bigcup_{j=1}^{\infty} F_{\beta_{j}}\left(Y^{\beta_{j}}\right) .
$$


We say that a sequence of open Riemannian manifolds $M_{j}$ has a glued limit space $Y$ if there exists a sequence $\delta_{i} \rightarrow 0$ satisfying the hypothesis of this theorem. A completed glued limit is defined to be the metric completion $\bar{Y}$ of a glued limit space $Y$, and the boundary of a glued limit space is defined to be the set $\bar{Y} \backslash Y$.

Remark 6.4. In Example 4.10, for sufficiently large $\delta_{0}$, each limit $Y^{\delta}$ is a ball in Euclidean 2-dimensional space. According to Theorem 6.3, the glued limit space of this sequence is constructed by taking the disjoint union of the ball $Y^{\delta_{0}}$ with the concentric annulus $Y^{\delta_{0} / i+1} \backslash Y^{\delta_{0} / i}$.

Remark 6.5. The definition of the glued limit space depends on the choice of $\delta_{i}$ and the isometric embeddings in Theorem 6.1. Even if one fixes the sequence $\delta_{i} \rightarrow 0$, the glued limit need not be unique; see Example 6.16.

Proof of Theorem 6.3. We first prove that $d_{Y}$ is positive definite. For the first and second cases of the definition of $d_{Y}$, we immediately see that $d_{Y}(x, y)=0$ if and only if $x=y$. For the third and fourth cases, notice that

$$
\varphi_{\delta_{i+j+1}, \delta_{i+1}}(y)=\left(\varphi_{\delta_{i+j+1}, \delta_{i+j}} \circ \varphi_{\delta_{i+j}, \delta_{i+1}}\right)(y)
$$

so

$$
\varphi_{\delta_{i+j+1}, \delta_{i+1}}(y) \in \varphi_{\delta_{i+j+1}, \delta_{i+j}}\left(Y^{\delta_{i+j}}\right) .
$$

Thus $x \neq \varphi_{\delta_{i+j+1}, \delta_{i+1}}(y)$ and

$$
d_{Y}(x, y)=d_{Y^{\delta_{i+j+1}}}\left(x, \varphi_{\delta_{i+j+1}, \delta_{i+1}}(y)\right) \neq 0 .
$$

Define $F_{\delta_{i}}: Y^{\delta_{i}} \rightarrow Y$ by

$$
F_{\delta_{i}}(y)= \begin{cases}y & \text { if } i=1, \\ y & \text { if } i>1 \text { and } y \in Y^{\delta_{i}} \backslash \varphi_{\delta_{i}, \delta_{i-1}}\left(Y^{\delta_{i-1}}\right) \\ \varphi_{\delta_{i}, \delta_{0}}^{-1}(y) & \text { if } i>1 \text { and } \varphi_{\delta_{i}, \delta_{0}}^{-1}(y) \in Y^{\delta_{0}}, \\ \varphi_{\delta_{i}, \delta_{j}}^{-1}(y) & \text { if } i>1 \text { and } \varphi_{\delta_{i}, \delta_{j}}^{-1}(y) \in Y^{\delta_{j}} \backslash \varphi_{\delta_{j}, \delta_{j-1}}\left(Y^{\delta_{j-1}}\right) \text { for some } j>1 .\end{cases}
$$

What we are doing in the third and fourth part of the definition of $F_{\delta_{i}}$ is the following. Suppose that $y \in Y^{\delta_{0} / i}$. Then either

$$
y \in Y^{\delta_{i}} \backslash \varphi_{\delta_{i}, \delta_{i-1}}\left(Y^{\delta_{i-1}}\right)
$$

or $y \in \varphi_{\delta_{i}, \delta_{i-1}}\left(Y^{\delta_{i-1}}\right)$. In the latter case, there exists $y_{i-1} \in Y^{\delta_{i-1}}$ such that $y=\varphi_{\delta_{i}, \delta_{i-1}}\left(y_{i-1}\right)$. If $i-1>1$, either

$$
y_{i-1} \in Y^{\delta_{i-1}} \backslash \varphi_{\delta_{i-1}, \delta_{i-2}}\left(Y^{\delta_{i-2}}\right) \quad \text { or } \quad y_{i-1} \in \varphi_{\delta_{i-1}, \delta_{i-2}}\left(Y^{\delta_{i-2}}\right) .
$$


Proceeding in the same way, if necessary, we find $j$ such that there exists $y_{j} \in Y^{\delta_{j}}$ satisfying the condition $y_{j} \notin \varphi_{\delta_{j}, \delta_{j-1}}\left(Y^{\delta_{j-1}}\right)$ if $j>1$, and also $y=\varphi_{\delta_{i}, \delta_{j}}\left(y_{j}\right)$.

It is easy to see that

$$
F_{\delta_{i}}\left(Y^{\delta_{i}}\right)=Y^{\delta_{0}} \cup \bigsqcup_{j=1}^{i-1}\left(Y^{\delta_{j+1}} \backslash \varphi_{\delta_{j+1}, \delta_{j}}\left(Y^{\delta_{j}}\right)\right)
$$

and for $j<i$,

$$
F_{\delta_{j}}=F_{\delta_{i}} \circ \varphi_{\delta_{i}, \delta_{j}}
$$

For arbitrary $\delta$, by Theorem 6.1 there exists a subsequence $M_{j_{k}}^{\delta}$ which converges in the Gromov-Hausdorff sense to a limit $Y^{\delta}$. Define $F_{\delta}: Y^{\delta} \rightarrow Y$ by

$$
F_{\delta}=F_{\left\{\delta,\left\{\delta_{i}\right\}\right\}}= \begin{cases}F_{\delta_{0}} \circ \varphi_{\delta_{0}, \delta} & \text { if } \delta_{0}<\delta, \\ F_{\delta_{i+1}} \circ \varphi_{\delta_{i+1}, \delta} & \text { if } \delta_{i+1} \leq \delta<\delta_{i},\end{cases}
$$

where $\varphi_{\delta_{0}, \delta}, \varphi_{\delta_{i+1}, \delta}$ are given in Theorem 6.1.

Observe that in the latter case of the definition of $F_{\delta}, F_{\delta_{i}}=F_{\delta} \circ \varphi_{\delta, \delta_{i}}$. This, together with the definition of $F_{\delta}$, gives

$$
F_{\delta_{i}}\left(Y^{\delta_{i}}\right) \subset F_{\delta}\left(Y^{\delta}\right) \subset F_{\delta_{i+1}}\left(Y^{\delta_{i+1}}\right) .
$$

Now that we have $\beta_{j}$ decreasing to 0 , there exists $N$ sufficiently large that $\beta_{j} \leq \delta_{0}$, and for all $j \geq N$, there exists $i$ such that $\beta_{j} \in\left[\delta_{i+1}, \delta_{i}\right)$. From (36) and (37), taking $\delta=\beta_{i}$, we conclude that

$$
Y=\bigcup_{j=N}^{\infty} F_{\beta_{j}}\left(Y^{\beta_{j}}\right)=\bigcup_{j=1}^{\infty} F_{\beta_{j}}\left(Y^{\beta_{j}}\right)
$$

because $F_{\beta_{0}}\left(Y^{\beta_{0}}\right) \subset F_{\beta_{N}}\left(Y^{\beta_{N}}\right)$.

To prove that $F_{\delta}$ is an isometric embedding, it is enough to prove that each $F_{\delta_{i}}$ is an isometric embedding. $F_{\delta_{0}}$ is an isometric embedding by definition of $Y$. For $F_{\delta_{i+1}}$ we must check three cases. Let $x, y \in Y^{\delta_{i+1}}$.

Case (i): If $x, y \in Y^{\delta_{i+1}} \backslash \varphi_{\delta_{i+1}, \delta_{i}}\left(Y^{\delta_{i}}\right)$, then $F_{\delta_{i+1}}(x)=x$, and $F_{\delta_{i+1}}(y)=y$.

Case (ii): If $x \in Y^{\delta_{i+1}} \backslash \varphi_{\delta_{i+1}, \delta_{i}}\left(Y^{\delta_{i}}\right)$ and $y \in \varphi_{\delta_{i+1}, \delta_{i}}\left(Y^{\delta_{i}}\right)$, then

$$
F_{\delta_{i+1}}(y)=\varphi_{\delta_{i+1}, \delta_{i+1-j}}^{-1}(y) \in Y^{\delta_{i+1-j}} \backslash \varphi_{\delta_{i+1-j}, \delta_{i-j}}\left(Y^{\delta_{i-j}}\right)
$$

for some $j$, so

$$
\begin{aligned}
d_{Y}\left(F_{\delta_{i+1}}(x), F_{\delta_{i+1}}(y)\right) & =d_{Y^{\delta_{i+1-j}}}\left(F_{\delta_{i+1}}(x), \varphi_{\delta_{i+1}, \delta_{i+1-j}}\left(F_{\delta_{i+1}}(y)\right)\right) \\
& =d_{Y^{\delta_{i+1}}}(x, y) .
\end{aligned}
$$


Case (iii): If $F_{\delta_{i+1}}(x)=\varphi_{\delta_{i+1}, \delta_{i+1-k}}^{-1}(x)$ and $F_{\delta_{i+1}}(y)=\varphi_{\delta_{i+1}, \delta_{i+1-j}}^{-1}(y)$, then take $k \leq j$. Recall that $\varphi_{\delta_{i+1}, \delta_{i+1-k}} \circ \varphi_{\delta_{i+1-k}, \delta_{i+1-j}}=\varphi_{\delta_{i+1}, \delta_{i+1-j}}$, so we have $d_{Y}\left(F_{\delta_{i+1}}(x), F_{\delta_{i+1}}(y)\right)$

$$
\begin{aligned}
& =d_{Y^{\delta_{i+1}-k}}\left(F_{\delta_{i+1}}(x), \varphi_{\delta_{i+1-k}, \delta_{i+1-j}}\left(F_{\delta_{i+1}}(y)\right)\right) \\
& =d_{Y^{\delta_{i+1}}}\left(\varphi_{\delta_{i+1}, \delta_{i+1-k}}\left(F_{\delta_{i+1}}(x)\right), \varphi_{\delta_{i+1}, \delta_{i+1-j}}\left(F_{\delta_{i+1}}(y)\right)\right) \\
& =d_{Y^{\delta_{i+1}}}(x, y) .
\end{aligned}
$$

The triangle inequality follows from the above paragraphs. For $x, y, z \in Y$, find $\delta$ such that $x, y, z \in F_{\delta}\left(Y^{\delta}\right)$. The triangle inequality holds for the preimages of $x, y, z$, and since $F_{\delta}$ is an isometric embedding, it also holds for $x, y, z$.

6C. Glued limits within Gromov-Hausdorff limits. Recall that in Theorem 4.3 we proved that if a sequence of open Riemannian manifolds $M_{j}$ has a GromovHausdorff limit $X$, then subsequences of the inner regions $M_{j}^{\delta}$ have GromovHausdorff limits. Here we assume that the $M_{j}$ also have a (possibly empty) completed glued limit space as in Theorem 6.3. We prove that this completed glued limit space is unique and provide a precise description as to how to find this completed glued limit space as a subset of the Gromov-Hausdorff limit (Theorem 6.6).

Note that the completed glued limit need not agree with the Gromov-Hausdorff limit (Remark 6.7). In fact, we provide an example where the completed glued limit space is empty (Remark 6.8).

It should be emphasized that we must assume the $M_{j}$ have a completed glued limit to obtain uniqueness. It is possible that a sequence $M_{j}$ has a Gromov-Hausdorff limit and that one needs a subsequence to obtain a glued limit, and that different subsequences provide different completed glued limits (see Remark 6.9).

Theorem 6.6. Let $\left\{M_{j}\right\}$ be a sequence of open manifolds that converges in the Gromov-Hausdorff sense to a compact metric space $\left(X, d_{X}\right)$. Suppose $Y$ is a glued limit space of the $\left\{M_{j}\right\}$ defined as in Theorem 6.3. Then the completed glued limit $\bar{Y}$ is isometric to the closure $\bar{U}_{\left\{j_{k}\right\}} \subset X$ of any limit's inner union $U_{\left\{j_{k}\right\}} \subset X$ defined as in Definition 4.7 for any subsequence $j_{k}$. In particular, any completed glued limit and the closure of any of the limit's inner regions are isometric.

We do not claim all the limit's inner regions are the same subset of $X$, and in fact this is not true, even after taking a closure. They are only isometric to one another. See Example 4.13.

Proof. Let $Y$ be a glued limit space defined using Theorems 6.3 and 6.1 via a sequence of isometric embeddings $\varphi_{j}$ of $M_{j}^{\delta_{i}} \subset M_{j}^{\delta_{i+1}}$ into a sequence of compact metric spaces $Z_{i}$ rather than a single compact metric space $Z$.

Since we have assumed the original sequence of Riemannian manifolds has a glued limit space $Y$ without requiring a subsequence, the following spaces are 
isometric:

$$
Y_{\left\{\delta_{i}\right\},\left\{j_{k}\right\}} \cong Y_{\left\{\delta_{i}\right\}} \cong Y_{\left\{\delta_{i}\right\},\left\{j_{k}^{\prime}\right\}}
$$

for any pair of subsequences $\left\{j_{k}\right\}$ and $\left\{j_{k}^{\prime}\right\}$.

Recall that Theorem 6.3 provides, for each $\delta>0$, an isometric embedding $F_{\delta}: Y^{\delta} \rightarrow Y$, with

$$
Y=\bigcup_{i=1}^{\infty} F_{\delta_{i}}\left(Y^{\delta_{i}}\right) \quad \text { and } \quad F_{\delta_{i}}\left(Y^{\delta_{i}}\right) \subset F_{\delta_{i+1}}\left(Y^{\delta_{i+1}}\right) .
$$

Since $Y^{\delta}$ is the Gromov-Hausdorff limit of the inner regions $M_{j}^{\delta}$, it is isometric to the limit of the inner regions $Y_{\left\{j_{k}\right\}}^{\delta} \subset U_{\left\{j_{k}\right\}} \subset X$ of Theorem 4.1. Note that we need a subsequence for each $\delta$ to produce the limit of the inner regions. We can produce a diagonal subsequence (also denoted $\left\{j_{k}\right\}$ ) such that

$$
Y_{\left\{j_{k}\right\}}^{\delta} \subset U_{\left\{j_{k}\right\}} \subset X \quad \text { is defined for all } \delta \in\left\{\delta_{i}\right\},
$$

so we have isometric embeddings

$$
\psi_{\delta_{i}}: F_{\delta_{i}}\left(Y^{\delta_{i}}\right) \subset Y \rightarrow Y_{\left\{j_{k}\right\}}^{\delta} \subset X .
$$

Since $F_{\delta_{i}}\left(Y^{\delta_{i}}\right) \subset F_{\delta_{i+1}}\left(Y^{\delta_{i+1}}\right)$, for each $i$ and any $h$ we may study the restriction

$$
\psi_{\delta_{i+h}}: F_{\delta_{i}}\left(Y^{\delta_{i}}\right) \subset Y \rightarrow Y_{\left\{j_{k}\right\}^{h}}^{\delta_{i}} \subset U_{\left\{j_{k}\right\}} \subset X .
$$

Since $F_{\delta_{i}}\left(Y^{\delta_{i}}\right)$ and $X$ are compact, we can find a subsequence $h_{k}$ depending on $i$ which converges to a limit isometric embedding:

$$
\psi_{i, \infty}: F_{\delta_{i}}\left(Y^{\delta_{i}}\right) \subset Y \rightarrow \bar{U}_{\left\{j_{k}\right\}} \subset X
$$

We may do this for each $i$ and diagonalize the subsequences if we wish. Since $\psi_{\delta_{i+h}}$ is a restriction of $\psi_{\delta_{i+1+h}}$, we see that $\psi_{i, \infty}$ is a restriction of $\psi_{i+1, \infty}$. Thus we may define an isometric embedding

$$
\psi_{\infty}: Y \rightarrow \bar{U}_{\left\{j_{k}\right\}} \subset X
$$

Extending this, we have an isometric embedding

$$
\bar{\psi}_{\infty}: \bar{Y} \rightarrow \bar{U}_{\left\{j_{k}\right\}}
$$

Since $X$ is compact, $\bar{U}_{\left\{j_{k}\right\}}$ is compact and thus so is $\bar{Y}$.

We need only construct an isometric embedding from $\bar{U}_{\left\{j_{k}\right\}}$ to $\bar{Y}$ to prove that these spaces are isometric, because they are compact metric spaces. We repeat the same trick as above but now use the fact that we have isometries

$$
F_{\delta_{i}}^{\prime}: Y_{\left\{j_{k}\right\}}^{\delta_{i}} \rightarrow F_{\delta_{i}}\left(Y^{\delta_{i}}\right) \subset Y
$$


and

$$
Y=\bigcup_{i=1}^{\infty} F_{\delta_{i}}^{\prime}\left(Y_{\left\{j_{k}\right\}}^{\delta_{i}}\right)
$$

with

$$
F_{\delta_{i}}^{\prime}\left(Y_{\left\{j_{k}\right\}}^{\delta_{i}}\right) \subset F_{\delta_{i+1}}^{\prime}\left(Y_{\left\{j_{k}\right\}}^{\delta_{i+1}}\right)
$$

Since $Y_{\left\{j_{k}\right\}}^{\delta_{i}} \subset Y^{\delta_{i+1}}\left(j_{k}\right)$, we may study for each $i$ and any $h$ the restriction

$$
F_{i+h}^{\prime}: Y_{\left\{j_{k}\right\}}^{\delta_{i}} \rightarrow Y \subset \bar{Y}
$$

Since we have shown $\bar{Y}$ is compact, a subsequence converges for each $i$ (and we can diagonalize these subsequences), so that we obtain isometric embeddings

$$
F_{i, \infty}^{\prime}: Y_{\left\{j_{k}\right\}}^{\delta_{i}} \rightarrow \bar{Y}
$$

Since $F_{i, \infty}^{\prime}$ is a restriction of $F_{i+1, \infty}^{\prime}$, we can define an isometric embedding

$$
F_{\infty}^{\prime}: U_{\left\{j_{k}\right\}} \rightarrow \bar{Y}
$$

This extends to an isometric embedding from $\bar{U}_{\left\{j_{k}\right\}}$ to $\bar{Y}$. Since we have a pair of isometric embeddings between a pair of compact metric spaces, these metric spaces are isometric.

Remark 6.7. It is possible that the completed glued limit is not the same as the Gromov-Hausdorff limit. Example 4.10 has a glued limit which is an open disk in Euclidean space; its completed glued limit is the closed disk, while its GromovHausdorff limit is a disk with a line segment attached.

Remark 6.8. The glued limit of a sequence of open Riemannian manifolds may exist but be the empty set. See, for example, the sequence $M_{2 j+1}$ in Example 4.11. This sequence converges in the Gromov-Hausdorff sense but $U$ is an empty set. It only satisfies the conditions of Theorem 6.1 in a trivial way: for each $\delta>0$ there exists $N_{\delta} \in \mathbb{N}$ such that $M_{j}^{\delta}=\varnothing$ for all $j \geq N_{\delta}$.

Remark 6.9. A sequence of $M_{j}$ which converges in the Gromov-Hausdorff sense may not have a glued limit space. In fact, one may need to take a subsequence to obtain a glued limit, and different subsequences might produce different glued limit spaces. In Examples 4.9-4.12, the subsequence $M_{2 j}$ has a completed glued limit space which is isometric to $\bar{U}_{\{2 j\}}$ and the subsequence $M_{2 j+1}$ has a completed glued limit space which is isometric to $\bar{U}_{\{2 j+1\}}$, but the sequence $M_{j}$ itself does not have a glued limit space. We thus see that the different glued limits obtained using different subsequences are quite different. In particular, in Example 4.11 the completed glued limit of the $M_{2 j}$ agrees with the Gromov-Hausdorff limit of the $M_{j}$, while the completed glued limit of the $M_{2 j+1}$ is empty. 
6D. Glued limit spaces when there are no Gromov-Hausdorff limits. In the setting of Theorem 6.1, the subsequence of manifolds $M_{j}$ such that $M_{j}^{\delta} \mathrm{GH} Y^{\delta}$ need not have any Gromov-Hausdorff limit. Here we discuss an old example and present two new examples.

Remark 6.10. The manifolds $M_{j}$ described in Example 2.13 have increasingly many spikes, and the sequence does not have a Gromov-Hausdorff limit. However, the sequence $M_{j}^{\delta}$ converges to the metric completion of the annulus $\operatorname{Ann}_{(0,0)}(1+\delta$, $2-\delta$ ) with the flat metric; see Remark 5.6. Start with $\delta_{0}<\frac{1}{2}$; then

$$
Y^{\delta_{0}}=\operatorname{Ann}_{(0,0)}\left(1+\delta_{0}, 2-\delta_{0}\right)
$$

and

$$
Y^{\delta_{0} /(i+1)} \backslash \varphi_{\delta_{0} /(i+1), \delta_{0} / i}\left(Y^{\delta_{0} / i}\right)=A_{1} \cup A_{2},
$$

where

$$
A_{1}=\operatorname{Ann}_{(0,0)}\left(1+\frac{\delta_{0}}{i+1}, 1+\frac{\delta_{0}}{i}\right) \quad \text { and } \quad A_{2}=\operatorname{Ann}_{(0,0)}\left(2-\frac{\delta_{0}}{i}, 2-\frac{\delta_{0}}{i+1}\right) .
$$

Thus $Y=\operatorname{Ann}_{(0,0)}(1,2)$ with the flat length metric. This glued limit space $Y$ is precompact.

A similar example, also constructed using flat manifolds $M_{j} \subset \mathbb{E}^{2}$ with no Gromov-Hausdorff limit, has convergent $M_{j}^{\delta}$ and a glued limit space which is a flat open manifold that is bounded but not precompact:

Example 6.11. We define a flat open manifold with $j$ spikes of decreasing width:

$$
M_{j}=U_{j} \cup V_{j},
$$

where

$$
\begin{aligned}
U_{j} & =\left\{(r \cos \theta, r \sin \theta): r<4+\sin \frac{4 \pi^{2}}{\theta}, \theta \in(2 \pi / j, 2 \pi]\right\}, \\
V_{j} & =\{(r \cos \theta, r \sin \theta): r<4, \theta \in(0,2 \pi / j]\} .
\end{aligned}
$$

As in Example 2.13, the $\left(M_{j}, d_{M_{j}}\right)$ have no Gromov-Hausdorff limit because they have increasingly many spikes. Unlike Example 2.13, for any number $N$, there exists $\delta_{N}$ sufficiently small such that $M_{j}^{\delta_{N}}$ has $N$ spikes. In fact,

$$
\left(M_{j}^{\delta}, d_{M_{j}}\right) \stackrel{\mathrm{GH}}{\longrightarrow}\left(Y^{\delta}, d_{Y}\right),
$$

where $Y^{\delta}$ is the $\delta$-inner region of the flat open manifold

$$
Y=\left\{(r \cos \theta, r \sin \theta): r<4+\sin \frac{4 \pi^{2}}{\theta}, \theta \in(0,2 \pi]\right\} .
$$

Taking the identity maps for the isometric embeddings, we see that $Y$ is also a glued limit space for the $M_{j}$, even though it is bounded but not precompact. 
Recall Example 2.14 of a sequence of surfaces having no Gromov-Hausdorff limit. We modify it to obtain a sequence of manifolds with boundary that has no Gromov-Hausdorff limit, but whose $\delta$-inner regions have Gromov-Hausdorff limits, and we construct the glued limit space and see that it is also bounded and not precompact. This glued limit space is not a manifold.

Example 6.12. Let

$$
X_{j}=([0,1] \times[0,1]) \sqcup\left([0,1] \times\left[0, \frac{1}{2}\right]\right) \sqcup \cdots \sqcup\left([0,1] \times\left[0,\left(\frac{1}{2}\right)^{j}\right]\right)
$$

be a disjoint union of spaces with taxicab metrics, glued via the map $\psi(0, y)=(0, y)$. One may think of $X_{j}$ as a book with $j$ pages of decreasing height glued along a spike on the left. Within $X_{j}$, choose $A_{j}$ to be the union of the top edges of each of the pages. If we take surfaces $M_{j}$ as constructed in Proposition 2.8, they now have boundary, and

$$
d_{\mathrm{GH}}\left(M_{j}, X_{j}\right) \rightarrow 0 \quad \text { and } \quad d_{\mathrm{GH}}\left(M_{j}^{\delta}, X_{j} \backslash T_{\delta}\left(A_{j}\right)\right) \rightarrow 0 .
$$

As in Example 2.14, the $M_{j}$ have no GH-convergent subsequence because the $X_{j}$ have no GH-convergent subsequence.

Observe that there exists $k_{\delta}$ such that, for all $j>k_{\delta}$,

$$
X_{j} \backslash T_{\delta}\left(A_{j}\right)=([0,1] \times[0, \delta)) \sqcup\left([0,1] \times\left[0, \frac{1}{2}-\delta\right]\right) \sqcup \cdots \sqcup\left([0,1] \times\left[0,\left(\frac{1}{2}\right)^{k_{\delta}}-\delta\right]\right) .
$$

Since this sequence does not depend on $j$, it clearly converges in the GromovHausdorff sense. Thus, the $M_{j}^{\delta}$ converge to the same Gromov-Hausdorff limit space. In fact, they converge to $X_{\infty} \backslash T_{\delta}\left(A_{\infty}\right)$, where

$$
X_{\infty}=([0,1] \times[0,1]) \sqcup\left([0,1] \times\left[0, \frac{1}{2}\right]\right) \sqcup \cdots \sqcup\left([0,1] \times\left[0,\left(\frac{1}{2}\right)^{j}\right]\right) \sqcup \cdots,
$$

and $A_{\infty}$ is the union of the tops of all of these pages. In fact, $X_{\infty}$ is the glued limit space.

6E. A glued limit space which is not geodesic. Here we present an example whose glued limit space is not geodesic or even a length space (and neither is its metric completion):

Example 6.13. In Euclidean space $\mathbb{E}^{2}$, define

$$
M_{j}=((-1,1) \times(-1,1)) \backslash\left(\left[-\frac{1}{2}, \frac{1}{2}\right] \times\left[0,1-\frac{1}{j}\right]\right) .
$$

Then, for $\delta<\frac{1}{4}$, there is $J=J(\delta)$ such that

$$
\begin{aligned}
M_{j}^{\delta}=((-1+\delta, 1-\delta) \times(-1+\delta,-\delta)) & \sqcup\left(\left(-1+\delta,-\frac{1}{2}-\delta\right) \times(-\delta, 1-\delta)\right) \\
& \sqcup\left(\left(\frac{1}{2}-\delta, 1-\delta\right) \times(-\delta, 1-\delta)\right)
\end{aligned}
$$

for $j \geq J$. 
Thus $\bar{M}_{j}^{\delta}$ is a constant sequence for $j \geq J$, and $\bar{M}_{j}^{\delta} \stackrel{\mathrm{GH}}{\longrightarrow} Y^{\delta}$, where $Y^{\delta}=[-1+\delta, 1-\delta] \times[-1+\delta, 0] \cup\left[-1+\delta,-\frac{1}{2}+\delta\right] \times[0,1-\delta]$

$$
\cup\left[\frac{1}{2}-\delta, 1-\delta\right] \times[0,1-\delta]
$$

The completed glued limit is not a length space:

$$
\bar{Y}=[-1,1] \times[-1,0] \cup\left[-1,-\frac{1}{2}\right] \times[0,1] \cup\left[\frac{1}{2}, 1\right] \times[0,1] \subset \mathbb{E}^{2} .
$$

Note that $\bar{M}_{j} \stackrel{\mathrm{GH}}{\longrightarrow} X=Y \cup\left(\{1\} \times\left[-\frac{1}{2}, \frac{1}{2}\right]\right)$.

Open question 6.14. Is a glued limit space locally geodesic: for all $y \in Y$, does there exist $\epsilon_{y}>0$ such that $B\left(y, \epsilon_{y}\right)$ is geodesic? If there is a counterexample, what conditions can be imposed on the space to guarantee that it is locally geodesic?

6F. Balls in glued limit spaces. Recall from Lemma 3.3 that for any $p \in M^{\delta_{i}}$, if $x \in B_{p}\left(\delta_{i}-\delta_{i+1}\right) \subset M$, then $x \in M^{\delta_{i+1}}$. This is not true for glued limit spaces. That is, it is possible for $p \in F_{\delta_{i}}\left(Y^{\delta_{i}}\right)$ to have an $x \in B_{p}\left(\delta_{i}-\delta_{i+1}\right) \subset Y$ such that $x \notin F_{\delta_{i+1}}\left(Y^{\delta_{i+1}}\right)$. In fact, we can take the ball of arbitrarily small radius and still have $x \notin F_{\delta_{i+1}}\left(Y^{\delta_{i+1}}\right)$ :

Example 6.15. In Example 6.12 we constructed a sequence $M_{j}$ having no GromovHausdorff limit, but such that the $M_{j}^{\delta}$ converge in the Gromov-Hausdorff sense to $Y^{\delta}=X_{\infty} \backslash T_{\delta}\left(A_{\infty}\right)$, where

$$
X_{\infty}=([0,1] \times[0,1]) \sqcup\left([0,1] \times\left[0, \frac{1}{2}\right]\right) \sqcup \cdots \sqcup\left([0,1] \times\left[0,\left(\frac{1}{2}\right)^{j}\right]\right) \sqcup \cdots,
$$

where each piece is connected along $(0, y) \sim(0, y)$ and $A_{\infty}$ is the union of the tops of all of these pages. This $X_{\infty}$ is a glued limit space for this example.

Then $F_{\delta}\left(Y^{\delta}\right)=X_{\infty} \backslash T_{\delta}\left(A_{\infty}\right)$. Take any ball about the common point $(0,0)$ in $X_{\infty}$. For any radius $r>0, B_{(0,0)}(r)$ contains infinitely many points

$$
y_{j}=\left(\frac{r}{2}, 0\right) \in[0,1] \times\left[0,\left(\frac{1}{2}\right)^{j}\right] .
$$

However, $y_{j} \notin F_{\delta}\left(Y^{\delta}\right)$ for $j$ sufficiently large that $\left(\frac{1}{2}\right)^{j}<\delta$.

6G. Nonuniqueness of the glued limit space. We now see that glued limit spaces and completed glued limit spaces are not necessarily unique. Recall that in Theorem 6.6 we explained that if the $M_{j}$ have a Gromov-Hausdorff limit, then the completed glued limit space is unique. So we need to construct a sequence of manifolds $M_{j}$ having no Gromov-Hausdorff limit. In fact, we will imitate Example 6.12, applying Proposition 2.8 to construct the following example:

Example 6.16. There are a sequence $\left\{M_{j}\right\}$ of Riemannian surfaces with boundary, a sequence $\delta_{i} \rightarrow 0$, and metric spaces $Y^{\delta_{i}}$ such that

$$
d_{\mathrm{GH}}\left(M_{j}^{\delta_{i}}, Y^{\delta_{i}}\right) \rightarrow 0
$$


with two different glued limit spaces

$$
Y_{1}=Y\left(\delta_{2 i}, \varphi_{\delta_{2 i}, \delta_{2 i+2}}\right) \quad \text { and } \quad Y_{2}=Y\left(\delta_{2 i}, \varphi_{\delta_{2 i}, \delta_{2 i+2}}^{\prime}\right),
$$

constructed as in Theorem 6.3 and Theorem 6.1, whose metric completions are not isometric.

Proof. Let

$$
P_{j}=[0,1] \times\left[-\frac{1}{2 j}, \frac{1}{2 j}\right] \text { for } j=1,2, \ldots,
$$

and let

$$
X_{j}=P_{1} \sqcup\left(P_{2} \sqcup P_{2}\right) \sqcup \cdots \sqcup\left(P_{j} \sqcup \cdots \sqcup P_{j}\right) \quad\left(2^{i-1} \text { copies of } P_{i}, 1 \leq i \leq j\right)
$$

be a disjoint union of $N_{j}=1+2+4+\cdots+2^{j-1}$ spaces endowed with taxicab metrics, glued via with the map $\psi(0, y)=(0, y)$. One may think of $X_{j}$ as a book with $N_{j}$ pages of different heights glued along a spike on the left.

Let $H_{j} \subset P_{j}$ be defined by

$$
H_{j}=[0,1] \times\left\{-\frac{1}{2 j}\right\} \cup\{1\} \times\left[-\frac{1}{2 j}, \frac{1}{2 j}\right] \cup[0,1] \times\left\{\frac{1}{2 j}\right\} \subset P_{j},
$$

and let $A_{j} \subset X_{j}$ be defined by

$$
A_{j}=H_{1} \sqcup\left(H_{2} \sqcup H_{2}\right) \sqcup \cdots \sqcup\left(H_{j} \sqcup \cdots \sqcup H_{j}\right) \quad\left(2^{i-1} \text { copies of } H_{i}, 1 \leq i \leq j\right) .
$$

If we take surfaces $M_{j}$ as constructed in Proposition 2.8, they now have a boundary, and we have

$$
d_{\mathrm{GH}}\left(M_{j}, X_{j}\right) \rightarrow 0 \quad \text { and } \quad d_{\mathrm{GH}}\left(M_{j}^{\delta}, X_{j} \backslash T_{\delta}\left(A_{j}\right)\right) \rightarrow 0 .
$$

As in Example 2.14, the $M_{j}$ have no GH-convergent subsequence because the $X_{j}$ have no GH-convergent subsequence.

Now

$$
\begin{aligned}
X_{j} \backslash T_{\delta}\left(A_{j}\right)=( & \left.P_{1} \backslash T_{\delta}\left(H_{1}\right)\right) \sqcup\left(P_{2} \backslash T_{\delta}\left(H_{2}\right)\right) \sqcup\left(P_{2} \backslash T_{\delta}\left(H_{2}\right)\right) \\
& \sqcup\left(P_{3} \backslash T_{\delta}\left(H_{3}\right)\right) \sqcup \cdots \sqcup\left(P_{3} \backslash T_{\delta}\left(H_{3}\right)\right) \\
& \vdots \\
& \sqcup\left(P_{j} \backslash T_{\delta}\left(H_{j}\right)\right) \sqcup \cdots \sqcup\left(P_{j} \backslash T_{\delta}\left(H_{j}\right)\right) .
\end{aligned}
$$

Observe that

$$
P_{j} \backslash T_{\delta}\left(H_{j}\right)=[0,1-\delta] \times\left[-\frac{1}{2 j}+\delta, \frac{1}{2 j}-\delta\right] .
$$

Taking $\delta=\delta_{2 i}=1 /(2 i)$ and $j>i$, we have

$$
P_{i} \backslash T_{\delta}\left(H_{i}\right)=\left[0,1-\frac{1}{2 i}\right] \times\{0\}
$$


and

$$
P_{j} \backslash T_{\delta}\left(H_{j}\right)=\varnothing
$$

Thus

$$
\begin{aligned}
X_{j} \backslash T_{\delta}\left(A_{j}\right)=( & \left.P_{1} \backslash T_{\delta}\left(H_{1}\right)\right) \sqcup\left(P_{2} \backslash T_{\delta}\left(H_{2}\right)\right) \sqcup\left(P_{2} \backslash T_{\delta}\left(H_{2}\right)\right) \\
& \sqcup\left(P_{3} \backslash T_{\delta}\left(H_{3}\right)\right) \sqcup \cdots \sqcup\left(P_{3} \backslash T_{\delta}\left(H_{3}\right)\right) \\
& \vdots \\
& \sqcup\left(P_{i-1} \backslash T_{\delta}\left(H_{i-1}\right)\right) \sqcup \cdots \sqcup\left(P_{i-1} \backslash T_{\delta}\left(H_{i-1}\right)\right) \\
& \sqcup\left[0,1-\frac{1}{2 i}\right] \times\{0\} \sqcup \cdots \sqcup\left[0,1-\frac{1}{2 i}\right] \times\{0\},
\end{aligned}
$$

endowed with taxicab metrics and glued together with the map $\psi(0, y)=(0, y)$. There are $1+2+4+\cdots+2^{(i-1)-1}$ rectangular pages and $2^{i-1}$ pages that are just intervals of length $1-1 /(2 i)$. Taking $j \rightarrow \infty$, we get

$$
d_{\mathrm{GH}}\left(X_{j} \backslash T_{\delta}\left(A_{j}\right), Y^{\delta}\right) \rightarrow 0,
$$

where

$$
Y^{\delta_{2 i}}=Y^{1 /(2 i)}=X_{j} \backslash T_{\delta_{2 i}}\left(A_{j}\right) \text { for all } j>i
$$

So

$$
\begin{aligned}
Y^{\delta_{2 i}}=( & \left.P_{1} \backslash T_{1 /(2 i)}\left(H_{1}\right)\right) \sqcup\left(P_{2} \backslash T_{1 /(2 i)}\left(H_{2}\right)\right) \sqcup\left(P_{2} \backslash T_{1 /(2 i)}\left(H_{2}\right)\right) \\
& \sqcup\left(P_{3} \backslash T_{1 /(2 i)}\left(H_{3}\right)\right) \sqcup \cdots \sqcup\left(P_{3} \backslash T_{1 /(2 i)}\left(H_{3}\right)\right) \\
& \vdots \\
& \sqcup\left(P_{2 i-1} \backslash T_{1 /(2 i)}\left(H_{2 i-1}\right)\right) \sqcup \cdots \sqcup\left(P_{2 i-1} \backslash T_{1 /(2 i)}\left(H_{2 i-1}\right)\right) \\
& \sqcup\left[0,1-\frac{1}{2 i}\right] \times\{0\} \sqcup \cdots \sqcup\left[0,1-\frac{1}{4 i}\right] \times\{0\},
\end{aligned}
$$

endowed with taxicab metrics and glued together with the map $\psi(0, y)=(0, y)$; there are $1+2+4+\cdots+2^{(i-1)-1}$ rectangular pages and $2^{i-1}$ pages that are just intervals of length $1-1 /(2 i)$.

If we define $\varphi_{\delta_{2 i}, \delta_{2 i+2}}: Y^{\delta_{2 i}} \rightarrow Y^{\delta_{2 i+2}}$ to be the inclusion map and then construct the glued limit space as in Theorem 6.1, we obtain

$$
\begin{aligned}
Y_{1}= & Y\left(\delta_{2 i}, \varphi_{\delta_{2 i}, \delta_{2 i+2}}\right)=Y \\
= & \left(P_{1} \backslash H_{1}\right) \sqcup\left(P_{2} \backslash H_{2}\right) \sqcup\left(P_{2} \backslash H_{2}\right) \\
& \sqcup\left(P_{3} \backslash H_{3}\right) \sqcup \cdots \sqcup\left(P_{3} \backslash H_{3}\right) \\
& \vdots \\
& \sqcup\left(P_{j} \backslash H_{j}\right) \sqcup \cdots \sqcup\left(P_{j} \backslash H_{j}\right) \cdots
\end{aligned}
$$


endowed with taxicab metrics glued with a gluing map $\psi(0, y)=(0, y)$. This has infinitely many pages, all shaped like rectangles.

Now we define $\varphi_{\delta_{2 i}, \delta_{2 i+2}}^{\prime}: Y^{\delta_{2 i}} \rightarrow Y^{\delta_{2 i+2}}$ to be an isometric embedding which maps a point

$$
(x, y) \in P_{k} \backslash T_{\delta_{2 i}}\left(H_{k}\right) \subset Y^{\delta_{2 i}}
$$

for $k<i$ to

$$
(x, y) \in P_{k} \backslash T_{\delta_{2 i+2}}\left(H_{k}\right) \subset Y^{\delta_{2 i+2}}
$$

via the inclusion map, and which maps

to

$$
(x, y) \in P_{i} \backslash T_{\delta_{2 i}}\left(H_{i}\right)=\left[0,1-\frac{1}{2 i}\right] \times\{0\} \subset Y^{\delta_{2 i}}
$$

$$
\left(x, y-\delta_{2 i}+\delta_{2 i+2}\right) \in P_{i+1} \backslash T_{\delta_{2 i+2}}\left(H_{i+1}\right)=\left[0,1-\frac{1}{2 i+2}\right] \times\{0\} \subset Y^{\delta_{2 i+2}} .
$$

This is possible because we have enough copies of $P_{i+1} \backslash T_{\delta_{2 i+2}}\left(H_{i+1}\right)$ in $Y^{\delta_{2 i+2}}$.

In particular, $\varphi_{\delta_{2 i}, \delta_{2 i+2}}^{\prime}$ maps the interval pages into interval pages. If we then construct the glued limit space as in Theorem 6.1, we obtain

$$
Y_{2}=Y\left(\delta_{2 i}, \varphi_{\delta_{2 i}, \delta_{2 i+2}}^{\prime}\right)=Y \sqcup[0,1] \times\{0\} \sqcup[0,1] \times\{0\} \sqcup[0,1] \times\{0\} \sqcup \cdots,
$$

which has infinitely many pages that are intervals in addition to all the pages shaped like rectangles. So we have two distinct glued limit spaces for the sequence $\delta_{2 i}=1 /(2 i)$, and their metric completions are not isometric.

\section{Glued limits under curvature bounds}

In this section we prove the existence of glued limits of sequences of manifolds with certain natural geometric conditions (Theorems 7.1 and 7.4). We do not require the sequences of manifolds themselves to have Gromov-Hausdorff limits.

7A. Constructing glued limits of manifolds with constant sectional curvature. In this section we prove that if $M_{j} \in \mathcal{M}_{H}^{m, V, l}$ for all $j$ (see Definition 5.1) then the sequence has a glued limit space (Theorem 7.1). The sequence need not have a Gromov-Hausdorff limit (see Remark 7.2).

Theorem 7.1. Given any $\delta_{0}>0$, if $\left(M_{j}, g_{j}\right) \subset \mu_{H}^{m, V, l}$, then there is a GromovHausdorff convergent subsequence $\left\{M_{j_{k}}^{\delta_{0}}\right\}$ and a glued limit space $Y$ such that for all $\delta \in\left(0, \delta_{0}\right]$ there exists a further subsequence $\left\{j_{k}^{\prime}\right\}$ of $\left\{j_{k}\right\}$ for which $M_{j_{k}^{\prime}}^{\delta}$ converges in the Gromov-Hausdorff sense to a compact metric space $Y^{\delta}$, and for any such $Y^{\delta}$ there exists an isometric embedding

$$
F_{\delta}: Y^{\delta} \rightarrow Y .
$$


Remark 7.2. The sequences of flat surfaces $M_{j} \subset \mathbb{E}^{2}$ defined in Example 2.13 and Example 6.11 have a common finite upper volume bound, but there is no common finite upper bound for the number of disjoint balls in $M_{j}$ of radius less than 1 . Thus, these two sequences do not have a Gromov-Hausdorff limit. Nonetheless, since

$$
L_{\min }\left(M_{j}\right)=\inf \left\{L_{g}(C): C \text { is a closed geodesic in } M_{j}\right\}>l,
$$

Theorem 7.1 demonstrates that we can construct glued limits for these spaces.

Remark 7.3. The choice of a further subsequence $\left\{j_{k}^{\prime}\right\}$ of $\left\{j_{k}\right\}$ in Theorem 7.1 is necessary. Let $\left(M_{j}, g_{j}\right) \subset M_{0}^{2, V, l}$ be the sequence defined in Example 4.4. Take $\delta_{0}=3 \varepsilon$. Then $\left\{M_{j}^{\delta_{0}}\right\}$ is a Gromov-Hausdorff convergent sequence. Choosing $2 \varepsilon \in\left(0, \delta_{0}\right]$, we see that $\bar{M}_{2 j}^{2 \varepsilon}$ converges in the Gromov-Hausdorff sense but $\bar{M}_{j}^{2 \varepsilon}$ does not.

Proof. Consider the sequence $\delta_{0}, \delta_{i}=\delta_{0} / i, i \in \mathbb{N}$. Start with $\delta_{0}$. By Theorem 5.2, there exist a sequence $\left\{\iota_{k}\left(\delta_{0}\right)\right\}$ of integers and a compact metric space $Y^{\delta_{0}}$ such that

$$
\left(\bar{M}_{\iota_{k}\left(\delta_{0}\right)}, d_{M_{\iota_{k}\left(\delta_{0}\right)}}\right) \stackrel{\mathrm{GH}}{\longrightarrow}\left(Y^{\delta_{0}}, d_{Y^{\delta_{0}}}\right) .
$$

Proceeding as before, for each $n \in \mathbb{N}$ there is a subsequence $\left\{\iota_{k}\left(\delta_{n}\right)\right\}_{k}$ of $\left\{\iota_{k}\left(\delta_{n-1}\right)\right\}_{k}$ and a compact metric space $Y_{\left\{\iota_{k}\left(\delta_{n}\right)\right\}}^{\delta_{n}}$ such that

$$
\left(\bar{M}_{\iota_{k}\left(\delta_{n}\right)}^{\delta_{n}}, d_{\left.M_{\iota_{k}\left(\delta_{n}\right)}\right)} \stackrel{\mathrm{GH}}{\longrightarrow} Y^{\delta_{n}} .\right.
$$

Define $j_{k}=\iota_{k}\left(\delta_{k}\right)$. We have

$$
\left(\bar{M}_{j_{k}}^{\delta_{n}}, d_{M_{j_{k}}}\right) \stackrel{\mathrm{GH}}{\longrightarrow} Y^{\delta_{n}}
$$

for $n=0,1,2, \ldots$ since $\left\{j_{k}\right\}_{k=n}^{\infty}$ is a subsequence of $\left\{\iota_{k}\left(\delta_{n}\right)\right\}_{k=1}^{\infty}$. We may now apply Theorem 6.3 to complete the proof.

7B. Constructing glued limits with Ricci curvature bounds. Here we prove that glued limits exist for noncollapsing sequences of manifolds with nonnegative Ricci curvature and bounded volume which have control on the intrinsic diameters of their inner regions (defined in (4)):

Theorem 7.4. Given $m \in \mathbb{N}$, a decreasing sequence $\delta_{i} \rightarrow 0,(i \geq 0), V>0$, $\theta>0$, and $D_{i}>0$, let $\left(M_{j}, g_{j}\right)$ be a sequence of m-dimensional open Riemannian manifolds with nonnegative Ricci curvature such that $\operatorname{Vol}\left(M_{j}\right) \leq V$,

and such that

$$
\sup \left\{\operatorname{Diam}\left(M_{j}^{\delta_{i}}, d_{M_{j}^{\delta_{i}}}\right): j \in \mathbb{N}\right\}<D_{i} \quad \text { for all } i \in \mathbb{N},
$$

$$
\text { for all } j \in \mathbb{N} \text {, there exists } q_{j} \in M_{j}^{\delta_{0}} \text { such that } \operatorname{Vol}\left(B_{q_{j}}\left(\delta_{0}\right)\right) \geq \theta \delta_{0}^{m} .
$$


Then there exists a sequence $\left\{j_{k}\right\} \rightarrow \infty$ such that for all $\delta_{i}$ the sequence $\left\{M_{j_{k}}^{\delta_{i}}\right\}$ converges in the Gromov-Hausdorff sense to a compact metric space $Y^{\delta_{i}}$. Thus, the $M_{j_{k}}$ have a glued limit space $Y$ such that for all $\delta \in\left(0, \delta_{0}\right]$ there is a further subsequence $\left\{j_{k}^{\prime}\right\}$ of $\left\{j_{k}\right\}$ for which $M_{j_{k}^{\prime}}^{\delta}$ converges in the Gromov-Hausdorff sense to a compact metric space $Y^{\delta}$ isometrically embedded in $Y$ :

$$
F_{\delta}: Y^{\delta} \rightarrow Y
$$

Remark 7.5. If there is $D>0$ such that

$$
\sup _{\delta \in\left(0, \delta_{0}\right]}\left\{\operatorname{Diam}\left(M_{j}^{\delta}, d_{M_{j}^{\delta}}\right)\right\} \leq D,
$$

then we could take $D_{i}=D$ for all $i$. But this requirement is unnecessarily strong.

Remark 7.6. The choice of a further subsequence $\left\{j_{k}^{\prime}\right\}$ of $\left\{j_{k}\right\}$ in Theorem 7.1 is necessary. For the sequence $\left(M_{j}, g_{j}\right)$ defined in Example 4.4, consider a decreasing sequence $\delta_{i} \rightarrow 0$ such that $\delta_{0}=3 \varepsilon$ and $\delta_{1}=\varepsilon$. Then the hypotheses of the theorem are satisfied. For all $\delta_{i},\left\{M_{j}^{\delta_{i}}\right\}$ converges in the Gromov-Hausdorff sense. However, for $2 \varepsilon \in\left(0, \delta_{0}\right],\left\{M_{j}^{2 \varepsilon}\right\}$ does not have a Gromov-Hausdorff limit.

Proof of Theorem 7.4. Take $\delta \in\left(0, \delta_{0}\right]$; by hypothesis and the Bishop-Gromov volume comparison (Theorem 2.19),

$$
\operatorname{Vol}\left(B_{q_{j}}(\delta)\right) \geq \operatorname{Vol}\left(B_{q_{j}}\left(\delta_{0}\right)\right)\left(\frac{\delta}{\delta_{0}}\right)^{m} \geq \theta \delta^{m} .
$$

This and the hypotheses of the theorem imply that, for each $i$,

$$
\left\{\left(M_{j}, g_{j}\right)\right\} \subset M_{\theta}^{m, \delta_{i}, D_{i}, V} .
$$

Start with $\delta_{0}$. By Theorem 1.4 there exists a sequence $\left\{\iota_{k}\left(\delta_{0}\right)\right\}$ of integers such that

$$
\left(\bar{M}_{\iota_{k}\left(\delta_{0}\right)}^{\delta_{0}}, d_{M_{\iota_{k}\left(\delta_{0}\right)}}\right) \stackrel{\mathrm{GH}}{\longrightarrow}\left(Y^{\delta_{0}}, d_{Y^{\delta_{0}}}\right) .
$$

Proceeding as before, for each $n \in \mathbb{N}$ there exists a subsequence $\left\{\iota_{k}\left(\delta_{n}\right)\right\}_{k}$ of $\left\{\iota_{k}\left(\delta_{n-1}\right)\right\}_{k}$ and a compact metric space $Y_{\left\{\iota_{k}\left(\delta_{n}\right)\right\}}^{\delta_{n}}$ such that

$$
\left(\bar{M}_{\iota_{k}\left(\delta_{n}\right)}^{\delta_{n}}, d_{M_{\iota_{k}\left(\delta_{n}\right)}}\right) \stackrel{\mathrm{GH}}{\longrightarrow}\left(Y^{\delta_{n}}, d_{Y \delta_{n}}\right) .
$$

Define $j_{k}=\iota_{k}\left(\delta_{k}\right)$. We have

$$
\left(\bar{M}_{j_{k}}^{\delta_{n}}, d_{M_{j_{k}}}\right) \stackrel{\mathrm{GH}}{\longrightarrow}\left(Y^{\delta_{n}}, d_{Y^{\delta_{n}}}\right)
$$

since $\left\{j_{k}\right\}_{k=n}^{\infty}$ is a subsequence of $\left\{\iota_{k}\left(\delta_{n}\right)\right\}_{k=1}^{\infty}$. Finally, apply Theorem 6.3. 


\section{Properties of glued limit spaces under curvature bounds}

In this final section of the paper we consider the local properties of the glued limits of sequences of manifolds with constant sectional curvature as in Theorem 7.1 and manifolds with nonnegative Ricci curvature as in Theorem 7.4. We begin with an example indicating how even when the sequences of manifolds have a GromovHausdorff limit, one need not retain curvature conditions on the Gromov-Hausdorff limit space (Example 8.1). This is in sharp contrast with the setting where the Riemannian manifolds are compact without boundary. In this example, the glued limit space is empty. Then we have a subsection about balls in glued limit spaces without any assumption on curvature (Theorem 8.3). We apply this control on the balls to prove that local curvature properties do persist on glued limit spaces. In particular, we prove (Proposition 8.4) that the glued limits of manifolds with constant sectional curvature bounds (and other conditions) are unions of manifolds with constant sectional curvature. We close with Theorem 8.8, concerning the metric measure properties of glued limits of manifolds with nonnegative Ricci curvature.

8A. An example with no curvature control. We now construct a sequence of flat open manifolds whose Gromov-Hausdorff limit is not flat:

Example 8.1. Let $B_{p}(1) \subset \mathbb{M}^{2}$ be a unit ball in hyperbolic space and $B_{0}(1) \subset \mathbb{E}^{2}$ be the unit ball in Euclidean space. Then $\exp _{p}: B_{0}(1) \rightarrow B_{p}(1)$. Let

$$
S_{j}=\left\{\left(\frac{i}{j}, \frac{k}{j}\right): i, k \in \mathbb{Z}\right\} \cap B_{0}(1) \subset \mathbb{E}^{2}
$$

and $S_{j}^{\prime}=\exp _{p}\left(S_{j}\right)$. Form a graph $A_{j}$ whose vertices are in $S_{j}$ and whose edges form a triangulation, by connecting $(i / j, k / j)$ to $((i+1) / j, k / j),(i / j,(k+1) / j)$ and $((i+1) / j,(k+1) / j)$. We let $A_{j}^{\prime}=\exp _{p}\left(A_{j}\right)$, and set the lengths of the edges in $A_{j}^{\prime}$ to be the distances between the vertices viewed as points in $\mathbb{H}^{2}$. Then $A_{j}^{\prime}$ converges to $B_{p}(1) \subset \mathbb{T}^{2}$ in the Gromov-Hausdorff sense.

Now define $A_{j}^{\prime \prime}$ to be the simplicial complex formed by filling in the triangles in $A_{j}^{\prime}$ with flat Euclidean triangles. Observe that $\left\{A_{j}^{\prime \prime}\right\}$ converges to $B_{p}(1) \subset \mathbb{T}^{2}$ in the Gromov-Hausdorff sense as well. Finally, for each $j$ we remove tiny balls of radius $\ll 1 / j$ around the vertices in $A_{j}^{\prime \prime}$, to create a flat open manifold $M_{j}$. These $M_{j}$ converge in the Gromov-Hausdorff sense to $B_{p}(1) \subset \mathbb{H}^{2}$.

Remark 8.2. Example 8.1 has an empty glued limit space. In the next subsections we will see that the glued limit spaces do retain some of the curvature properties of the initial sequence of manifolds. Thus the glued limit space is a more natural object of study than the Gromov-Hausdorff limit, even when the Gromov-Hausdorff limit exists. 
8B. Balls to glued limit spaces. Generally when one wishes to study the properties of a complete noncompact limit space, one studies balls in the limit space as Gromov-Hausdorff limits of balls in the sequence. Here we cannot control balls in the limit space, but we can control balls of radius $\epsilon<\delta_{i}-\delta_{i+1}$ centered in $F_{\delta_{i}}\left(Y^{\delta_{i}}\right)$ intersected with $F_{\delta_{i+1}}\left(Y^{\delta_{i+1}}\right)$. This will suffice to study the geometric properties of the glued limit spaces.

Theorem 8.3. Let $Y$ be a glued limit of a sequence $\left\{M_{j}\right\}$ of Riemannian manifolds, as in Theorem 6.3. If $y \in Y^{\delta_{i}}$ and $\epsilon<\delta_{i}-\delta_{i+1}$, then there exist a subsequence $\left\{M_{j_{k}}^{\delta_{i}}\right\}$ containing points $y_{j_{k}}$ and a sequence $\epsilon_{j_{k}} \rightarrow \epsilon$ such that

$$
B\left(y_{j_{k}}, \epsilon_{j_{k}}\right)=\left\{x \in M_{j_{k}}: d_{M}\left(x, y_{j_{k}}\right)<\epsilon_{j_{k}}\right\} \subset M_{j_{k}}^{\delta_{i+1}}
$$

and

$$
d_{\mathrm{GH}}\left(\left(\bar{B}\left(y_{j_{k}}, \epsilon_{j_{k}}\right), d_{M_{j_{k}}}\right),\left(\bar{B}\left(F_{\delta_{i}}(y), \epsilon\right) \cap F_{\delta_{i+1}}\left(Y^{\delta_{i+1}}\right), d_{Y}\right)\right) \rightarrow 0 .
$$

Note that in Example 6.15 we saw that $B\left(F_{\delta_{i}}(y), \epsilon\right) \cap F_{\delta_{i+1}}\left(Y^{\delta_{i+1}}\right)$ need not be isometric to $B\left(F_{\delta_{i}}(y), \epsilon\right) \subset Y$, even when $\epsilon$ is taken arbitrarily small.

Proof. Recall that in Theorem 6.1 we found $\varphi_{\delta_{i+1}, \delta_{i}}$ defined in the following way. We picked isometric embeddings

$$
\varphi_{j}: M_{j}^{\delta_{i+1}} \rightarrow Z \quad \text { and } \quad \varphi_{\infty}: Y^{\delta_{i+1}} \rightarrow Z
$$

such that

$$
d_{H}^{Z}\left(\varphi_{j}\left(M_{j}^{\delta_{i+1}}\right), \varphi_{\infty}\left(Y_{j}^{\delta_{i+1}}\right)\right) \rightarrow 0 .
$$

Then we found a subsequence such that

$$
d_{H}^{Z}\left(\varphi_{j_{k}}\left(M_{j_{k}}^{\delta_{i}}\right), X^{\delta_{i}}\right) \rightarrow 0
$$

and chose $\varphi_{\delta_{i+1}, \delta_{i}}$ to be an isometry such that

$$
\varphi_{\delta_{i+1}, \delta_{i}}\left(Y^{\delta_{i}}\right)=\varphi_{\infty}^{-1}\left(X^{\delta_{i}}\right)
$$

Then there exist

$$
y_{j_{k}} \in M_{j_{k}}^{\delta_{i}} \subset M_{j_{k}}^{\delta_{i+1}} \subset M_{j_{k}}
$$

such that

$$
d_{Z}\left(\varphi_{j_{k}}\left(y_{j_{k}}\right), \varphi_{\infty}\left(\varphi_{\delta_{i+1}, \delta_{i}}(y)\right) \rightarrow 0 .\right.
$$

Let $\epsilon^{\prime} \in\left(0, \delta_{i}-\delta_{i+1}\right)$. Then by Lemma 3.3 we have

$$
B\left(y_{j_{k}}, \epsilon^{\prime}\right)=\left\{x \in M_{j_{k}}: d_{M}\left(x, y_{j_{k}}\right)<\epsilon^{\prime}\right\} \subset M_{j_{k}}^{\delta_{i+1}} .
$$

From this, and since $\varphi_{j_{k}}: M_{j_{k}}^{\delta_{i+1}} \rightarrow Z$ is an isometry into its image, we see that $\left(B\left(y_{j_{k}}, \epsilon^{\prime}\right), d_{M_{j_{k}}}^{\delta_{i+1}}\right) \quad$ is isometric to $\left(B\left(\varphi_{j_{k}}\left(y_{j_{k}}\right), \epsilon^{\prime}\right) \cap \varphi_{j_{k}}\left(M_{j_{k}}^{\delta_{i+1}}\right), d_{Z}\right)$. 
By Lemma 2.2, for any $\epsilon \in\left(0, \delta_{i}-\delta_{i+1}\right)$, there exists $\epsilon_{j_{k}} \rightarrow \epsilon$ eventually in $\left(0, \delta_{i}-\delta_{i+1}\right)$, such that

$$
\bar{B}\left(\varphi_{j_{k}}\left(y_{j_{k}}\right), \epsilon_{j_{k}}\right) \cap \varphi_{j_{k}}\left(M_{j_{k}}^{\delta_{i+1}}\right) \stackrel{\mathrm{H}}{\longrightarrow} \bar{B}\left(\varphi_{\infty} \varphi_{\delta_{i+1}, \delta_{i}}(y), \epsilon\right) \cap \varphi_{\infty}\left(Y^{\delta_{i+1}}\right) .
$$

Now,

$$
\left(\bar{B}\left(\varphi_{\infty} \varphi_{\delta_{i+1}, \delta_{i}}(y), \epsilon\right) \cap \varphi_{\infty}\left(Y^{\delta_{i+1}}\right), d_{Z}\right)
$$

is isometric to

$$
\left(\bar{B}\left(\varphi_{\delta_{i+1}, \delta_{i}}(y), \epsilon\right), d_{Y^{\delta_{i+1}}}\right)
$$

which is isometric to

$$
\left(F_{\delta_{i+1}} \bar{B}\left(\varphi_{\delta_{i+1}, \delta_{i}}(y), \epsilon\right), d_{F_{\delta_{i+1}}\left(Y^{\delta_{i+1}}\right)}\right)
$$

which is isometric to

$$
\left(\bar{B}\left(F_{\delta_{i+1}} \varphi_{\delta_{i+1}, \delta_{i}}(y), \epsilon\right) \cap F_{\delta_{i+1}} Y^{\delta_{i+1}}, d_{Y}\right) .
$$

Hence

$$
d_{\mathrm{GH}}\left(\left(\bar{B}\left(y_{j_{k}}, \epsilon_{j_{k}}\right), d_{M_{j_{k}}}\right),\left(\bar{B}\left(F_{\delta_{i+1}}\left(\varphi_{\delta_{i+1}, \delta_{i}}(y)\right), \epsilon\right) \cap F_{\delta_{i+1}} Y^{\delta_{i+1}}, d_{Y}\right)\right) \rightarrow 0 .
$$

8C. Properties of glued limits of manifolds with constant sectional curvature. Here we prove a proposition, present a key example and state two open questions concerning the glued limits of manifolds with constant sectional curvature.

Proposition 8.4. Let $Y$ be a glued limit space obtained as in Theorem 7.1 from a sequence $M_{j} \in M_{H}^{m, V, l}$. Then there exists a countable collection of sets $W_{i} \subset Y$, each of which is isometric to an m-dimensional smooth open manifold of constant sectional curvature $H$, such that

In fact,

$$
Y \subset \bigcup_{i=1}^{\infty} W_{i}
$$

$$
F_{\delta_{i}}\left(Y^{\delta_{i}}\right) \subset W_{i} \subset F_{\delta_{i+1}}\left(Y^{\delta_{i+1}}\right) \subset Y .
$$

See Example 8.5, in which the glued limit space is a countable collection of flat tori which are not connected to one another but have a metric restricted from a larger compact metric space of finite volume.

Proof. Recall that any glued limit space $Y$ defined as in Theorem 6.3 depends on a sequence $\delta_{i} \rightarrow 0$ and gluings $\varphi_{\delta_{i+1}, \delta_{i}}: Y^{\delta_{i+1}} \rightarrow Y^{\delta_{i}}$ via the subsequential limit isometric embeddings of (32). There are isometric embeddings $F_{\delta_{i}}: Y^{\delta_{i}} \rightarrow Y$ such that

$$
Y \subset \bigcup_{i=1}^{\infty} F_{\delta_{i}}\left(Y^{\delta_{i}}\right)
$$


and

$$
F_{\delta_{i}}\left(Y^{\delta_{i}}\right) \subset F_{\delta_{i+1}}\left(Y^{\delta_{i+1}}\right)
$$

Let

$$
\epsilon_{i}=\frac{1}{2} \min \left\{\delta_{i}-\delta_{i+1}, \frac{l}{2}, \frac{\pi \sqrt{h}}{2}\right\},
$$

where $h=H$ when $H>0$ and $h=(l / \pi)^{2}$ otherwise.

Let

$$
W_{i}=T_{\epsilon_{i}}\left(F_{\delta_{i}}\left(Y^{\delta_{i}}\right)\right) \cap F_{\delta_{i+1}}\left(Y^{\delta_{i+1}}\right) \subset Y .
$$

First observe that by (54) we have

$$
F_{\delta_{i}}\left(Y^{\delta_{i}}\right) \subset W_{i}
$$

So combined with (53), we have (52). So we need only show $W_{i}$ is a smooth $m$-dimensional open manifold of constant sectional curvature $H$.

For all $w \in W_{i}$, there exists $y_{\infty} \in F_{\delta_{i}}\left(Y^{\delta_{i}}\right)$ such that $w \in B_{y_{\infty}}\left(\epsilon_{i}\right) \subset Y$. Since

$$
B_{y_{\infty}}\left(\epsilon_{i}\right) \subset T_{\epsilon_{i}}\left(F_{\delta_{i}}\left(Y^{\delta_{i}}\right)\right)
$$

we have

$$
U=B_{y_{\infty}}\left(\epsilon_{i}\right) \cap F_{\delta_{i+1}}\left(Y^{\delta_{i+1}}\right)=B_{y_{\infty}}\left(\epsilon_{i}\right) \cap W_{i} .
$$

We need only show that $U$ is isometric to a ball of radius $\epsilon_{i}$ in $M_{H}^{m}$, the $m$ dimensional simply connected manifold with constant sectional curvature $H$.

There exists $y \in Y^{\delta_{i}}$ such that $y_{\infty}=F_{\delta_{i}}(y)$. By Theorem 8.3, and the fact that $\epsilon_{i}<\delta_{i}-\delta_{i+1}$, there exists a subsequence $M_{j_{k}}^{\delta_{i}}$ containing points $y_{j_{k}}$ and $\epsilon_{j_{k}} \rightarrow \epsilon_{i}$ such that (50) and (51) are satisfied.

Since $\epsilon_{i}<l / 2$, we have $\epsilon_{j_{k}}<l / 2$ for $k$ sufficiently large, and so by (50) the $M_{j}$ satisfy the conditions of Theorem 5.2, and by (27) we know there is a Riemannian isometric diffeomorphism from $B\left(y_{j_{k}}, \epsilon_{j_{k}}\right)$ to a ball in $M_{H}^{m}$, the $m$ dimensional simply connected manifold with constant sectional curvature $H$. Since $\epsilon_{i}<\sqrt{H} \pi / 2$ when $H>0$, we have a convex ball, so that, as metric spaces,

$$
\left(B\left(y_{j_{k}}, \epsilon_{j_{k}}\right), d_{M}\right) \text { is isometric to }\left(B\left(p, \epsilon_{j_{k}}\right), d_{M_{H}^{m}}\right) .
$$

Taking $k \rightarrow \infty$, the closures of these latter balls converge in the GromovHausdorff sense to $\left(\bar{B}\left(p, \epsilon_{i}\right), d_{M_{H}^{m}}\right)$. Thus, by (51) and the uniqueness of GH limits,

$$
\left(\bar{B}\left(y_{\infty}, \epsilon_{i}\right) \cap F_{\delta_{i+1}}\left(Y^{\delta_{i+1}}\right), d_{Y}\right) \text { is isometric to }\left(\bar{B}\left(p, \epsilon_{i}\right), d_{M_{H}^{m}}\right) .
$$

Thus we have (55), and we are done.

Example 8.5. In this example we construct a glued limit space $Y$ for a sequence of manifolds $M_{j}^{m}$ satisfying the conditions of Theorem 5.2. In addition, the $M_{j}^{m}$ converge in the Gromov-Hausdorff sense to a metric space $X$, so that the glued 
limit space is unique. The glued limit $Y$ is a countable union of connected flat manifolds with the restricted metric from $X$.

Proof. Let $M_{1}$ be two flat square annuli connected by a slanted strip of width 1 and length $\sqrt{2}$ :

$$
M_{1}=C_{0,1} \cup C_{1,1} \cup S_{0,1} \subset \mathbb{R}^{3},
$$

where

$$
\begin{aligned}
& C_{0,1}=\left(((-1,1) \times(-1,1)) \backslash\left(\left(-\frac{1}{2}, \frac{1}{2}\right) \times\left[-\frac{1}{2}, \frac{1}{2}\right]\right)\right) \times\{0\}, \\
& C_{1,1}=\left(((-1,1) \times(-1,1)) \backslash\left(\left(-\frac{1}{2}, \frac{1}{2}\right) \times\left[-\frac{1}{2}, \frac{1}{2}\right]\right)\right) \times\{1\}, \\
& S_{0,1}=\left\{(x, y, z):(x, y) \in\left(-\frac{1}{2}, \frac{1}{2}\right) \times\left[-\frac{1}{2}, \frac{1}{2}\right], z=x+\frac{1}{2}\right\} .
\end{aligned}
$$

Endowed with the length metric, this is isometric to an open manifold with constant sectional curvature 0 . Note that, for $\delta>\frac{1}{4}$,

$$
M_{1}^{\delta} \subset C_{0,1} \cup C_{1,1} .
$$

Let $M_{2}$ be three flat square annuli of total area at most $4+4+4\left(\frac{1}{4}\right)$ connected by two slanted strips of width $\frac{1}{2}$ :

$$
M_{2}=C_{0,2} \cup C_{1,2} \cup C_{2,2} \cup S_{1,2} \cup S_{2,2} \subset \mathbb{R}^{3},
$$

where

$$
\begin{aligned}
& C_{0,2}=\left(((-1,1) \times(-1,1)) \backslash\left(\left(-\frac{1}{4}, \frac{1}{4}\right) \times\left[-\frac{1}{4}, \frac{1}{4}\right]\right)\right) \times\{0\}, \\
& C_{1,2}=\left(\left(\left(-\frac{1}{2}, \frac{1}{2}\right) \times\left(-\frac{1}{2}, \frac{1}{2}\right)\right) \backslash\left(\left(-\frac{1}{4}, \frac{1}{4}\right) \times\left[-\frac{1}{4}, \frac{1}{4}\right]\right)\right) \times\left\{\frac{1}{2}\right\}, \\
& C_{2,2}=\left(((-1,1) \times(-1,1)) \backslash\left(\left(-\frac{1}{4}, \frac{1}{4}\right) \times\left[-\frac{1}{4}, \frac{1}{4}\right]\right)\right) \times\left\{\frac{2}{2}\right\}, \\
& S_{0,2}=\left\{(x, y, z):(x, y) \in\left(-\frac{1}{4}, \frac{1}{4}\right) \times\left[-\frac{1}{4}, \frac{1}{4}\right], z=x+\frac{1}{4}\right\}, \\
& S_{1,2}=\left\{(x, y, z):(x, y) \in\left(-\frac{1}{4}, \frac{1}{4}\right) \times\left[-\frac{1}{4}, \frac{1}{4}\right], z=x+\frac{3}{4}\right\} .
\end{aligned}
$$

Endowed with the length metric, this is isometric to an open manifold with constant sectional curvature 0 . Note that, for $\delta>\frac{1}{8}$,

$$
M_{1}^{\delta} \subset C_{0,2} \cup C_{1,2} \cup C_{2,2} \backslash(B((0,0), \delta) \times[0,1]) .
$$

Let $M_{j}$ be $(j+1)$ flat square annuli of total area at most $4+4 \sum_{i=0}^{j}\left(\frac{1}{2}\right)^{j}$, connected by $j$ slanted strips of width $\left(\frac{1}{2}\right)^{j}$ :

$$
M_{j}=\bigcup_{i=0}^{j} C_{i, j} \cup \bigcup_{i=0}^{j-1} S_{i, j} \subset \mathbb{R}^{3},
$$

where, with the notation 
$I_{k}=\left(-\left(\frac{1}{2}\right)^{k},\left(\frac{1}{2}\right)^{k}\right), \quad \bar{I}_{k}=\left[-\left(\frac{1}{2}\right)^{k},\left(\frac{1}{2}\right)^{k}\right], \quad$ and $\quad m_{j}=\frac{2^{i+1-j}-2^{i-j}}{\left(\frac{1}{2}\right)^{j+1}-\left(-\frac{1}{2}\right)^{j+1}}$,

we define

$$
\begin{aligned}
C_{0, j} & =\left(\left(I_{0} \times I_{0}\right) \backslash\left(I_{j+1} \times \bar{I}_{j+1}\right)\right) \times\{0\}, \\
C_{i, j} & =\left(\left(I_{j-i} \times I_{j-i}\right) \backslash\left(I_{j+1} \times \bar{I}_{j+1}\right)\right) \times\left\{2^{i-j}\right\}, \\
C_{j, j} & =\left(\left(I_{0} \times I_{0}\right) \backslash\left(I_{j+1} \times \bar{I}_{j+1}\right)\right) \times\left\{2^{j-j}\right\}, \\
S_{0, j} & =\left\{(x, y, z):(x, y) \in I_{j+1} \times \bar{I}_{j+1}, z=x+\left(\frac{1}{2}\right)^{j+1}\right\}, \\
S_{i, j} & =\left\{(x, y, z):(x, y) \in I_{j+1} \times \bar{I}_{j+1}, z=m_{j}\left(x+\left(\frac{1}{2}\right)^{j+1}\right)+2^{i-j}\right\} .
\end{aligned}
$$

Endowed with the length metric, this is isometric to an open manifold with constant sectional curvature 0 . Note that, for $\delta>\left(\frac{1}{2}\right)^{j+2}$,

$$
M_{j}^{\delta} \subset C_{0, j} \cup \cdots \cup C_{j, j} \backslash(B((0,0), \delta) \times[0,1]) .
$$

The Gromov-Hausdorff limit of the $M_{j}$ exists and can be see to be

$$
X=\bigcup_{j=0}^{\infty} C_{j} \cup S_{0} \subset \mathbb{R}^{3},
$$

where

$$
\begin{aligned}
& C_{0}=I_{0} \times I_{0} \times\{0\}, \\
& C_{i}=I_{j-i} \times I_{j-i} \times\left\{2^{i-j}\right\}, \\
& S_{0}=\{0\} \times\{0\} \times[0,1],
\end{aligned}
$$

endowed with the length metric. The Gromov-Hausdorff limit $Y^{\delta}$ of the $M_{j}^{\delta}$ exists, and

$$
Y^{\delta} \subset X \backslash(B((0,0), \delta) \times[0,1])
$$

In fact, $Y=X \backslash S_{0}$.

Open question 8.6. Are the glued limits of sequences of manifolds with constant sectional curvature open manifolds with constant sectional curvature? We know they need not be connected by Example 8.5.

Open question 8.7. Are the glued limits of sequences of manifolds with constant sectional curvature unique? Perhaps an adaptation of Example 8.5 could be applied to show that they are not.

8D. Properties of glued limits of manifolds with nonnegative Ricci curvature. We now prove the final theorem of our paper and state the last two open questions: Theorem 8.8. Suppose that we have a sequence of $m$-dimensional open Riemannian manifolds $M_{j}$ with nonnegative Ricci curvature and $\operatorname{Vol}\left(M_{j}\right) \leq V_{0}$, and there 
exists a sequence $\delta_{i} \rightarrow 0$ such that the inner regions $M_{j}^{\delta_{i}}$ converge in the GromovHausdorff sense as $j \rightarrow \infty$ to $Y^{\delta_{i}}$ without collapsing. Suppose that $Y$ is a glued limit constructed as in Theorem 6.3. Then $Y$ has Hausdorff dimension $m, \mathscr{H}^{m}(Y) \leq V_{0}$, and its Hausdorff measure has positive lower density everywhere.

Note that this theorem may be applied to study the glued limits of sequences of manifolds satisfying the conditions of Theorem 7.4.

To prove this theorem we will apply Cheeger and Colding's volume convergence theorem [3; 4], which was reviewed in Section 2F. See Theorem 2.25 and Remark 2.26 for the precise statement we will use here.

Proof. First we prove that

$$
W_{i}=T_{\left(\delta_{i}-\delta_{i+1}\right) / 2}\left(F_{\delta_{i}}\left(Y^{\delta_{i}}\right)\right) \cap F_{\delta_{i+1}}\left(Y^{\delta_{i+1}}\right) \subset Y
$$

have Hausdorff dimension $m$ and have doubling Hausdorff measures. For any $w \in W_{i}$, let

$$
U_{w}=B\left(w, \frac{\delta_{i}-\delta_{i+1}}{2}\right) \cap W_{i} .
$$

We can find $y \in Y^{\delta_{i}}$ such that $d_{Y}(y, w)<\left(\delta_{i}-\delta_{i+1}\right) / 2$. Then we have

$$
U_{w}=B\left(w, \frac{\delta_{i}-\delta_{i+1}}{2}\right) \cap B\left(F_{\delta_{i}}(y), \delta_{i}-\delta_{i+1}\right) \cap F_{\delta_{i+1}}\left(Y^{\delta_{i+1}}\right) .
$$

By Theorem 8.3, we have a sequence $\left\{j_{k}\right\}$, points $y_{j_{k}} \in M_{j_{k}}^{\delta_{i}}$, and a sequence $\left\{\epsilon_{j_{k}}\right\} \rightarrow \epsilon=\left(\delta_{i}-\delta_{i+1}\right) / 2$ satisfying (50) and (51):

$$
d_{\mathrm{GH}}\left(\left(\bar{B}\left(y_{j_{k}}, \epsilon_{j_{k}}\right), d_{M_{j_{k}}}\right),\left(\bar{B}\left(F_{\delta_{i}}(y), \epsilon\right) \cap F_{\delta_{i+1}}\left(Y^{\delta_{i+1}}\right), d_{Y}\right)\right) \rightarrow 0 .
$$

Combining this with the fact that

$$
w \in B\left(y, \frac{\delta_{i}-\delta_{i+1}}{2}\right) \subset \bar{B}\left(F_{\delta_{i}}(y), \epsilon\right) \cap F_{\delta_{i+1}}\left(Y^{\delta_{i+1}}\right) \subset Y,
$$

there exist

$$
z_{j, k} \in \bar{B}\left(y_{j_{k}}, \frac{\delta_{i}-\delta_{i+1}}{2}\right) \subset \bar{B}\left(y_{j_{k}}, \epsilon_{j_{k}}\right) \subset M_{j_{k}}
$$

such that

$$
d_{\mathrm{GH}}\left(\left(\bar{B}\left(z_{j_{k}}, \frac{\delta_{i}-\delta_{i+1}}{2}\right), d_{M_{j_{k}}}\right),\left(\bar{U}_{w}, d_{Y}\right)\right) \rightarrow 0 .
$$

Since we assumed this is noncollapsing, then by the Cheeger-Colding volume convergence theorem mentioned above we have

$$
\mathscr{H}_{m}\left(B_{w}(r) \cap U_{w}\right)=\lim _{k \rightarrow \infty} \mathscr{H}_{m}\left(B_{z_{j_{k}}}(r)\right)
$$

for all $r \leq r_{i}=\left(\delta_{i}-\delta_{i+1}\right) / 2$. By (58) and Bishop's volume comparison theorem, we see that

$$
\mathscr{H}_{m}\left(B_{w}(r) \cap W_{i}\right)=\mathscr{H}_{m}\left(B_{w}(r) \cap U_{w}\right) \leq \omega_{m} r^{m} \text { for all } r \leq r_{i}
$$


is positive and finite for any $w \in W_{i}$. By the Bishop-Gromov volume comparison theorem,

$$
\frac{\mathscr{H}_{m}\left(B_{w}\left(r_{1}\right) \cap W_{i}\right)}{\mathcal{H}_{m}\left(B_{w}\left(r_{2}\right) \cap W_{i}\right)} \geq \frac{r_{1}^{m}}{r_{2}^{m}} \quad \text { for all } w \in W_{i}, r_{1}<r_{2} \leq r_{i}
$$

Since $W_{i}$ is a subset of the compact $F_{\delta_{i+1}}\left(Y^{\delta_{i+1}}\right)$, it is precompact. Choose a maximal collection $\left\{w_{1}, \ldots, w_{N}\right\} \subset W_{i}$ such that the $B\left(w_{i}, r_{i} / 2\right)$ are disjoint. Then

$$
W_{i} \subset \bigcup_{n=1}^{N} B\left(w_{n}, r_{i}\right)
$$

and

$$
\mathscr{H}^{m}\left(W_{i}\right) \leq \sum_{n=1}^{N} \mathscr{H}^{m}\left(B\left(w_{n}, r_{i}\right) \leq\left(\frac{1}{4}\right)^{m} \sum_{n=1}^{N} \mathscr{H}^{m}\left(B\left(w_{n}, r_{i} / 4\right)\right) .\right.
$$

But it is not hard to see, examining (50), that $B\left(w_{n}, r_{i} / 2\right)$ are the limits of disjoint balls in $M_{j}$, so

$$
\sum_{n=1}^{N} \mathscr{H}^{m}\left(B\left(w_{n}, r_{i} / 4\right)\right) \leq \limsup _{j \rightarrow \infty} \mathscr{H}_{m}\left(M_{j}^{\delta_{i}}\right) \leq V_{0} .
$$

So $W_{i}$ has Hausdorff dimension $m$ and

$$
\mathscr{H}_{m}\left(W_{i}\right) \leq V_{0} .
$$

Now

$$
Y=\bigcup_{i=1}^{\infty} W_{i}
$$

so it has Hausdorff dimension $m$ and

$$
\mathscr{H}_{m}(Y) \leq V_{0}
$$

Now to see that $Y$ has positive density everywhere, we must show

$$
\Theta_{*}\left(y, \mathscr{H}^{m}\right)=\liminf _{r \rightarrow 0} \frac{\mathscr{H}_{m}(B(y, r))}{r^{m}}>0 .
$$

For fixed $i \geq I_{y}$, we have

$$
\mathscr{H}_{m}(B(y, r)) \geq \mathscr{H}_{m}\left(B(y, r) \cap W_{i}\right) .
$$


Combining this with (59), we have

$$
\begin{aligned}
\Theta_{*}\left(y, \mathscr{H}^{m}\right) & =\liminf _{r \rightarrow 0} \frac{\mathscr{H}_{m}\left(B(y, r) \cap W_{i}\right)}{r^{m}} \\
& \geq \liminf _{r \rightarrow 0} \frac{\mathscr{H}_{m}\left(B\left(y, r_{i}\right) \cap W_{i}\right)}{r_{i}^{m}} \\
& \geq \frac{\mathscr{H}_{m}\left(B\left(y, r_{i}\right) \cap W_{i}\right)}{r_{i}^{m}}>0 .
\end{aligned}
$$

Open question 8.9. Are glued limit spaces of sequences as in Theorem 8.8 unique?

Open question 8.10. Are glued limit spaces of sequences as in Theorem 8.8 countably $\mathscr{H}^{m}$-rectifiable?

\section{Acknowledgements}

We would like to thank Stephanie Alexander (UIUC) for informing us about the work of Wong and Kodani when we first began to explore the question. We'd like to thank Frank Morgan (Williams) and David Johnson (Lehigh) for their interest and encouragement. We'd like to thank Pedro Solórzano (UC Riverside) for looking over some of the proofs. Thanks to Tabitha (IS 25), Penelope (IS 25) and Kendall (PS 32) for building the models of Examples 1.1-1.2 depicted in Figures 1, 2 and 5 and for computing the areas of the manifolds in Example 1.1 as part of a $\mathrm{K}-12$ outreach. Finally we would like to thank Jorge Basilio (CUNY), Christine Briener (MIT), Maria Hempel (ETH Zurich), Sajjad Lakzian (CUNY), Christopher Lonke (Carnegie Mellon), Mike Munn (University of Missouri at Columbia), Jacobus Portegies (Courant, NYU), and Timothy Susse (CUNY) for actively participating with us in the CUNY Metric Geometry Reading Seminar in the summer of 2012 and Kenneth Knox (Stony Brook) for joining us in the spring of 2013.

\section{References}

[1] Michael Anderson, Atsushi Katsuda, Yaroslav Kurylev, Matti Lassas, and Michael Taylor. Boundary regularity for the Ricci equation, geometric convergence, and Gel'fand's inverse boundary problem. Invent. Math., 158(2):261-321, 2004.

[2] Dmitri Burago, Yuri Burago, and Sergei Ivanov. A course in metric geometry. Number 33 in Graduate Studies in Mathematics. American Mathematical Society, Providence, RI, 2001.

[3] Jeff Cheeger and Tobias Holck Colding. On the structure of spaces with Ricci curvature bounded below, I. J. Differential Geom., 46(3):406-480, 1997.

[4] Tobias Holck Colding. Ricci curvature and volume convergence. Ann. of Math. (2), 145(3):477501, 1997.

[5] Tobias Holck Colding and Aaron Naber. Characterization of tangent cones of noncollapsed limits with lower Ricci bounds and applications. Geom. Funct. Anal., 23(1):134-148, 2013. 
[6] Manfredo Perdigão do Carmo. Geometria Riemanniana. Number 10 in Projeto Euclides. Instituto de Matemática Pura e Aplicada, Rio de Janeiro, 2nd edition, 1988. Translated in Riemannian Geometry, Birkhäuser, Boston, 1992.

[7] Mikhael Gromov. Groups of polynomial growth and expanding maps. Inst. Hautes Études Sci. Publ. Math., 53:53-73, 1981.

[8] Mikhael Gromov. Structures métriques pour les variétés Riemanniennes. CEDIC, Paris, 1981. Translated in Metric structures for Riemannian and non-Riemannian spaces, Birkhäuser, Boston, 2007.

[9] W. Klingenberg. Contributions to Riemannian geometry in the large. Ann. of Math. (2), 69:654666, 1959.

[10] Kenneth S. Knox. A compactness theorem for Riemannian manifolds with boundary and applications. preprint, 2013.

[11] Shigeru Kodani. Convergence theorem for Riemannian manifolds with boundary. Compositio Math., 75(2):171-192, 1990.

[12] Raquel Perales. A survey on the convergence of manifolds with boundary. preprint, 2013.

[13] Christina Sormani and Guofang Wei. Hausdorff convergence and universal covers. Trans. Amer. Math. Soc., 353(9):3585-3602, 2001.

[14] Jeremy Wong. An extension procedure for manifolds with boundary. Pacific J. Math., 235(1):173199, 2008

Received February 8, 2013. Revised June 18, 2013.

RAQUel PERALES

DEPARTMENT OF MATHEMATICS

STONY BROOK UNIVERSITY

100 NICOLLS RD

STONY BROOK, NY 11794

UNITED STATES

praquel@math.sunysb.edu

CHRISTINA SORMANI

DEPARTMENT OF MATHEMATICS

CUNy Graduate Center and Lehman College

365 FIFTH AVENUE

NEW YORK, NY 10016

UNITED STATES

sormanic@member.ams.org 


\title{
PACIFIC JOURNAL OF MATHEMATICS
}

\author{
msp.org/pjm
}

Founded in 1951 by E. F. Beckenbach (1906-1982) and F. Wolf (1904-1989)

\section{EDITORS}

Don Blasius (Managing Editor)

Department of Mathematics

University of California

Los Angeles, CA 90095-1555

blasius@math.ucla.edu

\author{
Paul Balmer \\ Department of Mathematics \\ University of California \\ Los Angeles, CA 90095-1555 \\ balmer@math.ucla.edu \\ Robert Finn \\ Department of Mathematics \\ Stanford University \\ Stanford, CA 94305-2125 \\ finn@math.stanford.edu \\ Sorin Popa \\ Department of Mathematics \\ University of California \\ Los Angeles, CA 90095-1555 \\ popa@math.ucla.edu
}

\author{
Vyjayanthi Chari \\ Department of Mathematics \\ University of California \\ Riverside, CA 92521-0135 \\ chari@math.ucr.edu \\ Kefeng Liu \\ Department of Mathematics \\ University of California \\ Los Angeles, CA 90095-1555 \\ liu@math.ucla.edu \\ Jie Qing \\ Department of Mathematics \\ University of California \\ Santa Cruz, CA 95064 \\ qing@ cats.ucsc.edu
}

\section{PRODUCTION}

Silvio Levy, Scientific Editor, production@msp.org

\section{SUPPORTING INSTITUTIONS}

ACADEMIA SINICA, TAIPEI

CALIFORNIA INST. OF TECHNOLOGY

INST. DE MATEMÁTICA PURA E APLICADA

KEIO UNIVERSITY

MATH. SCIENCES RESEARCH INSTITUTE

NEW MEXICO STATE UNIV.

OREGON STATE UNIV.

\author{
STANFORD UNIVERSITY \\ UNIV. OF BRITISH COLUMBIA \\ UNIV. OF CALIFORNIA, BERKELEY \\ UNIV. OF CALIFORNIA, DAVIS \\ UNIV. OF CALIFORNIA, LOS ANGELES \\ UNIV. OF CALIFORNIA, RIVERSIDE \\ UNIV. OF CALIFORNIA, SAN DIEGO \\ UNIV. OF CALIF., SANTA BARBARA
}

\author{
Daryl Cooper \\ Department of Mathematics \\ University of California \\ Santa Barbara, CA 93106-3080 \\ cooper@math.ucsb.edu \\ Jiang-Hua Lu \\ Department of Mathematics \\ The University of Hong Kong \\ Pokfulam Rd., Hong Kong \\ jhlu@maths.hku.hk \\ Paul Yang \\ Department of Mathematics \\ Princeton University \\ Princeton NJ 08544-1000 \\ yang@math.princeton.edu
}

These supporting institutions contribute to the cost of publication of this Journal, but they are not owners or publishers and have no responsibility for its contents or policies.

See inside back cover or msp.org/pjm for submission instructions.

The subscription price for 2014 is US $\$ 410 /$ year for the electronic version, and \$535/year for print and electronic.

Subscriptions, requests for back issues and changes of subscribers address should be sent to Pacific Journal of Mathematics, P.O. Box 4163, Berkeley, CA 94704-0163, U.S.A. The Pacific Journal of Mathematics is indexed by Mathematical Reviews, Zentralblatt MATH, PASCAL CNRS Index, Referativnyi Zhurnal, Current Mathematical Publications and Web of Knowledge (Science Citation Index).

The Pacific Journal of Mathematics (ISSN 0030-8730) at the University of California, c/o Department of Mathematics, 798 Evans Hall \#3840, Berkeley, CA 94720-3840, is published twelve times a year. Periodical rate postage paid at Berkeley, CA 94704, and additional mailing offices. POSTMASTER: send address changes to Pacific Journal of Mathematics, P.O. Box 4163, Berkeley, CA 94704-0163.

PJM peer review and production are managed by EditFLOW ${ }^{\circledR}$ from Mathematical Sciences Publishers.

\section{PUBLISHED BY}

\section{mathematical sciences publishers \\ nonprofit scientific publishing}

http://msp.org/

(C) 2014 Mathematical Sciences Publishers 


\section{PACIFIC JOURNAL OF MATHEMATICS}

Volume $270 \quad$ No. $2 \quad$ August 2014

Disjointification inequalities in symmetric quasi-Banach spaces and 257 their applications

SERgey Astashinin, Fedor A. SukocheV and DMitriy

ZANIN

Hamiltonian evolutions of twisted polygons in parabolic manifolds:

287

The Lagrangian Grassmannian

GLORIA MARÍ BEFFA

On Schwarz-Christoffel mappings

MARTIN CHUAQUi and CHRISTIAN POMMERENKE

Vanishing viscosity in the plane for nondecaying velocity and vorticity, II

ELAINE COZZI

Affine quantum Schur algebras and affine Hecke algebras

QIANG FU

On the classification of Killing submersions and their isometries

JosÉ M. MANZANO

Locally Lipschitz contractibility of Alexandrov spaces and its applications

Ayato Mitsuishi and TAKaO Yamaguchi

Sequences of open Riemannian manifolds with boundary

RAQUEL PERALES and CHRISTINA SORMANI

Invariant differential operators on a class of multiplicity-free spaces 